\title{
Learning-Based Joint Super-Resolution and Deblocking for a Highly Compressed Image
}

\author{
Li-Wei Kang, Member, IEEE, Chih-Chung Hsu, Boqi Zhuang, Chia-Wen Lin, Senior Member, IEEE, and \\ Chia-Hung Yeh, Senior Member, IEEE
}

\begin{abstract}
A highly compressed image is usually not only of low resolution, but also suffers from compression artifacts (blocking artifact is treated as an example in this paper). Directly performing image super-resolution (SR) to a highly compressed image would also simultaneously magnify the blocking artifacts, resulting in an unpleasing visual experience. In this paper, we propose a novel learning-based framework to achieve joint single-image SR and deblocking for a highly-compressed image. We argue that individually performing deblocking and SR (i.e., deblocking followed by SR, or SR followed by deblocking) on a highly compressed image usually cannot achieve a satisfactory visual quality. In our method, we propose to learn image sparse representations for modeling the relationship between low- and high-resolution image patches in terms of the learned dictionaries for image patches with and without blocking artifacts, respectively. As a result, image SR and deblocking can be simultaneously achieved via sparse representation and morphological component analysis (MCA)-based image decomposition. Experimental results demonstrate the efficacy of the proposed algorithm.
\end{abstract}

Index Terms-Dictionary learning, image decomposition, image super-resolution, morphological component analysis (MCA), selflearning, sparse representation.

\section{INTRODUCTION}

W ITH the rapid development of multimedia and network technologies, delivering and sharing multimedia contents over the Internet and heterogeneous devices has become more and more popular. However, due to limited channel band-

Manuscript received March 25, 2014; revised November 10, 2014 and February 17, 2015; accepted May 10, 2015. Date of publication May 15, 2015; date of current version June 13, 2015. This work was supported in part by the Taiwan Ministry of Science and Technology under Grant MOST 101-2221-E007-121-MY3, Grant 103-2221-E-007-046-MY3, and Grant MOST 103-2221E-224-034-MY2. The associate editor coordinating the review of this manuscript and approving it for publication was Dr. Shahram Shirani.

L.-W. Kang is with the Graduate School of Engineering Science and Technology-Doctoral Program and the Department of Computer Science and Information Engineering, National Yunlin University of Science and Technology, Yunlin 64002, Taiwan.

C.-C. Hsu is with the Department of Electrical Engineering, National Tsing Hua University, Hsinchu 30013, Taiwan.

B. Zhuang is with the Altek Corporation, Hsinchu 30078, Taiwan.

C.-W. Lin is with the Department of Electrical Engineering and the Institute of Communications Engineering, National Tsing Hua University, Hsinchu 30013, Taiwan, and with the Department of Computer Science and Information Engineering, Asia University, Taichung 41354, Taiwan (e-mail: cwlin@ee.nthu. edu.tw).

C.-H. Yeh is with the Department of Electrical Engineering, National Sun Yat-Sen University, Kaohsiung 80424, Taiwan.

Color versions of one or more of the figures in this paper are available online at http://ieeexplore.ieee.org.

Digital Object Identifier 10.1109/TMM.2015.2434216 width and storage capability, most images distributed over the Internet exist in low-quality versions degraded from the sources. The most common image degradations come from downscaling and compression. Downscaling reduces the spatial resolution in an image, whereas compression further reduces the redundancy in the spatial and temporal domains. Although downscaling together with compression can greatly reduce the required bandwidth and storage for images, they would also lead to significant information loss and unpleasing visual artifacts, including blocking, ringing, or blurring [1].

\section{A. Image Super-Resolution}

There has been a great demand for improving the perceptual quality of images in terms of the spatial resolution enhancement of an image, also known as image super-resolution (SR). The goal of image SR is to recover a high-resolution (HR) image from one or multiple low-resolution (LR) input images, which is essentially an ill-posed inverse problem [2]. There are mainly two categories of approaches for image SR: (i) traditional approaches and (ii) exemplar/learning-based approaches. In the traditional approaches, one sub-category is reconstruction-based schemes, where a set of LR images of the same scene are aligned with sub-pixel accuracy to generate a HR image [3]. Such kind of approaches mainly rely on multi-frame alignment, which is usually time-consuming and inaccurate, and cannot be used for single-image SR since it requires multiple input LR images. The other sub-category is frame interpolation [4], which has been shown to generate overly smooth images with ringing and jaggy artifacts.

The exemplar/learning-based methods [5]-[14] hallucinate the high frequency details of a LR image based on the co-occurrence prior between LR and HR image patches in a training set, which has proven to provide much finer details compared to the traditional approaches. More specifically, for a LR input, exemplar-based methods [5]-[8] search for similar image patches from a pre-collected training LR image dataset or the same image itself based on self-examples, and use their corresponding HR versions to produce the final SR output. Nevertheless, the fine details reconstructed by such exemplar-based methods, though looking visually similar, cannot be guaranteed to provide the true missing HR details. Hence, the performance of this approach highly relies on the similarity between the training set and test set or the self-similarity in the image itself.

Moreover, learning-based SR approaches [9]-[14] focus on modeling the relationship between different resolutions of images. For example, Yang et al. [9] proposed to apply sparse 
coding techniques to learn a compact representation for HR/LR patch pairs for SR based on pre-collected HR/LR image pairs. Then, Yang et al. [10] advances [9] to propose a coupled dictionary training approach for SR based on patch-wise sparse recovery, where the learned couple dictionaries relate the HR/LR image patch spaces via sparse representations. It is guaranteed that the sparse representation of a LR image patch can well reconstruct its underlying HR image patch, which cannot be guaranteed in [9]. Moreover, a sparse representation based framework was proposed in [11] for image deblurring and SR based on adaptive sparse domain selection and adaptive regularization, where two adaptive regularization terms are introduced. In addition, Ren et al. [12] proposed to utilize context-aware sparsity prior to enhance the performance of sparsity-based restoration approach for image denoising and SR. This method mitigates the artifacts in the produced HR images based on incorporating the structural correlations of dictionary atoms into the employed sparse coding algorithm. On the other hand, self-learning frameworks based on self-similarity of an image were introduced for SR in [13] and [14].

\section{B. Motivation of SR for a Highly Compressed Image}

The above-mentioned SR approaches, however, only consider that an input LR image is only degraded by down-scaling or at most an additional blurring operation (with a known or well-estimated blurring kernel). It is not always practical in a network environment, where image compression is usually adopted, which yields additional compression artifacts such as blocking and ringing. For image search engines, compression helps reduce the image size by up to $50 \%$ without obvious perceptual quality loss presented in the LR form of an image. Nevertheless, if SR is directly performed on the compressed LR image, compression artifacts will be simultaneously magnified and therefore the perceptual quality of resulting HR image would be poor [15]. Hence, a high-performance SR scheme for highly-compressed images is desirable for enhancing the resolution of image/video degraded by both down-scaling and compression. In [15], a unified framework was proposed to simultaneously improve the resolution and perceptual quality of low-quality web image/video degraded by down-scaling and compression. This approach combines adaptive regularization and learning-based SR, where the regularization strength is determined by the JPEG compression quality factor (QF) of an input image. QF is an integer ranging from 0 and 100 , which controls the degree of compression for JPEG images. The larger the $\mathrm{QF}$ is, the lower the compression ratio and the higher the image quality are [24].

On the other hand, an exemplar-based SR algorithm of compressed videos in DCT (discrete cosine transform) domain was proposed in [16]. The inputs to the system include a compressed LR video together with a HR still image of similar content which is assumed to be available in advance. In addition, a unified framework achieving simultaneous denoising and SR for noisy video was proposed in [17]. This approach assesses the visual quality with respect to fidelity preservation, detail preservation, and spatio-temporal smoothness. More works for low-quality video SR can be found in [18] and [19].

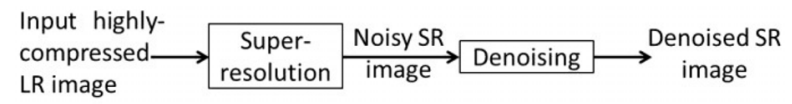

(a)

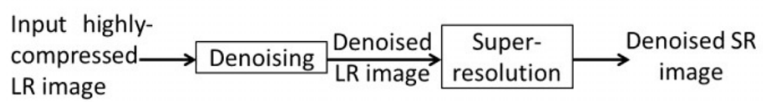

(b)

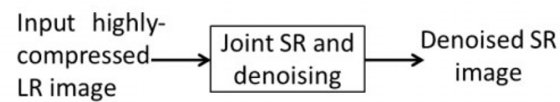

(c)

Fig. 1. SR methods for a highly compressed image: (a) cascading structure I: SR followed by denoising; (b) cascading structure II: denoising followed by SR; and (c) joint SR and denoising.

Existing SR approaches for low-quality images/videos [15]-[19], however, are all designed for some special purposes. For example, the SR framework proposed in [15] is mainly designed for JPEG compressed web images with known QFs. Moreover, most other related approaches are designed for video [16]-[19]. In addition, strictly speaking, most existing SR methods for low-quality images usually rely on two cascading structures: 1) SR followed by a denoising operation [cascading structure I in Fig. 1(a), and Fig. 2] denoising followed by SR [cascading structure II in Fig. 1(b)]. In the first cascading structure, the SR operation would significantly magnify the compression artifacts [e.g., the magnified blocking artifacts in Fig. 2(c)], making it difficult to eliminate the magnified artifacts using the next denoising operation. On the other hand, the second cascading structure (i.e., denoising followed by SR), would inevitably lose some image details caused by denoising (e.g., deblocking), thereby degrading the performance of SR, as exemplified in Fig. 2(d). Therefore, a joint SR and denoising structure [see Fig. 1(c)] is desirable as it not only avoids magnifying compression artifact but also reconstructs finer image details so as to achieve good-quality SR even the input image is highly compressed.

To achieve joint SR and deblocking, we proposed in the preliminary conference version [23] of this paper a self-learningbased SR framework to achieve joint SR and blocking artifact removal for a single LR image. The proposed method self-learns from the input image itself the sparse representations for modeling the relationship between LR and HR image patches based on the dictionaries learned from image patches with and without blocking artifacts, respectively. Based on the learned sparse representations, MCA (morphological component analysis)-based image decomposition [27], [28] is then applied to decompose and remove blocking artifacts so as to achieve image SR and deblocking simultaneously.

\section{Contribution of Proposed Method}

It should be noted that most research works on pure SR usually simulate the production of an input LR image as two degradation steps. For example, in [9], [10], it is assumed that an observed LR image is a blurred and down-sampled version of its HR version. In addition, the work proposed in [11] considers a more general image restoration problem, where an observed LR image is a blurred and down-sampled version of its HR version 


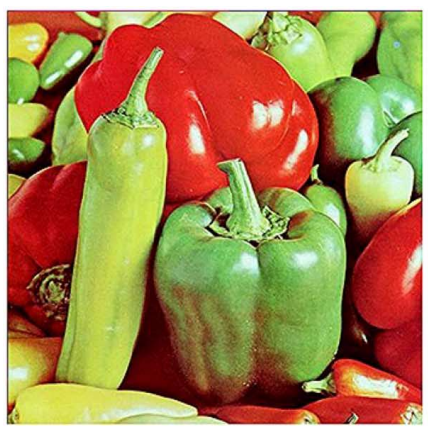

(a)

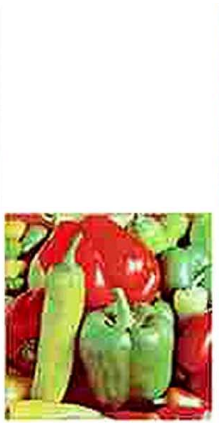

(b)

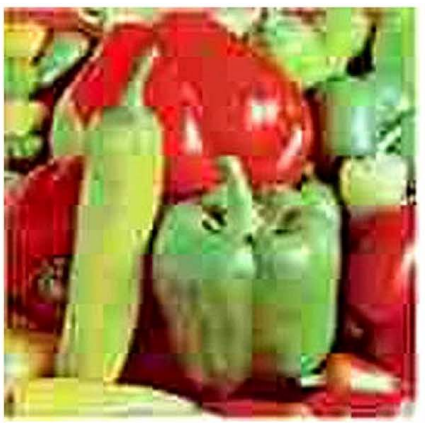

(c)

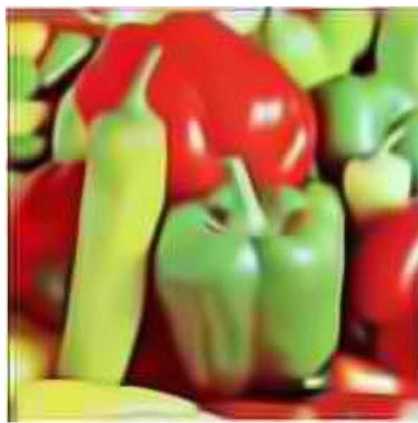

(d)

Fig. 2. Example illustrating the main problems caused by existing SR approaches for a highly compressed image: (a) the original HR image; (b) a LR and highly compressed version of (a); the SR version of (a) obtained by (c) directly applying the bicubic interpolation; and (d) applying image deblocking first, followed by SR (the Cascading-based approach used for comparison with the proposed method, described in Section IV). It can be observed from (c) that the blocking artifacts are significantly magnified by SR, while from (d) that some details are blurred by deblocking, resulting in poor SR result.

with additional additive noise. Moreover, the context-aware approach in [12] was proposed for LR images with Gaussian noise of known standard deviation. However, the image artifacts (e.g., additive Gaussian noise or blurring effect) considered in these works are significantly different from blocking artifact considered in this paper.

In our SR framework, we focus on a single input LR image/video frame being highly compressed by a block-based image/video compression algorithm (e.g., JPEG [24], H.264/AVC [25], or HEVC [26]), which has been widely used in current multimedia applications (e.g., images from WWW or video frames from YouTube ${ }^{1}$ ). To this end, we propose a learning-based sparse representation framework to achieve joint single-image SR and deblocking for a highly compressed image. Blocking artifact is one of the major visually unpleasing artifacts introduced by transform block-based compression and hence, deblocking has been extensively studied in the literature [1], [20]-[22]. The main contribution of this paper is three-fold: i) to the best of our knowledge, we are among the first to propose a unified framework for joint blocking artifact removal and single-image SR via sparse coding-based image decomposition; ii) the proposed framework can be adapted to any block transform-based compressed image/video without the need of any prior knowledge about the data source, coding bitrates, and compression algorithms; and iii) the proposed framework can be easily integrated into existing self-learning-based SR approaches [13], [14], where no extra training samples are required, or non-self-learning-based ones [5]-[12], which require additional training samples collected offline.

Compared with the preliminary conference version [23], this paper has been significantly extended in the following aspects: (i) In this paper, we further extend the proposed framework to non-self-learning-based SR approaches which was not discussed in [23]. (ii) We conduct subjective quality evaluations based on paired comparisons for different SR and deblocking schemes. (iii) This paper provides in-depth analyses and interpretations about the experimental results and computational complexity to offer good insights about the proposed method.

\footnotetext{
${ }^{1}$ [Online]. Available: http://www.youtube.com/
}

The rest of this paper is organized as follows. Section II reviews some related works. Section III presents the proposed SR framework for highly compressed images. In Section IV, experimental results are demonstrated. Finally, Section V concludes this paper.

\section{PRELIMINARIES}

In this section, we briefly review the concept of the general MCA-based framework for image decomposition and the utilized sparse representation and dictionary learning techniques. The major idea of MCA is to utilize the morphological diversity of different features contained in the data to be decomposed and to associate each morphological component to a dictionary of atoms. The MCA-based image decomposition technique described below will be integrated in the proposed SR framework mainly for image deblocking.

\section{A. MCA-Based Image Decomposition}

Suppose an image $I$ of $N$ pixels is a superposition of $S$ layers (called morphological components), denoted by $I=\sum_{s=1}^{S} I_{s}$, where $I_{s}$ denotes the $s$-th component, such as the geometric or textural component of $I$. To decompose image $I$ into $\left\{I_{s}\right\}_{s=1}^{S}$, the MCA algorithms [22], [27]-[30] iteratively minimize the following energy function:

$$
E\left(\left\{I_{s}\right\}_{s=1}^{S},\left\{\theta_{s}\right\}_{s=1}^{S}\right)=\frac{1}{2}\left\|I-\sum_{s=1}^{S} I_{s}\right\|_{2}^{2}+\tau \sum_{s=1}^{S} E_{s}\left(I_{s}, \theta_{s}\right)
$$

where $\theta_{s}$ denotes the sparse coefficients corresponding to $I_{s}$ with respect to dictionary $D_{s}, \tau$ is a regularization parameter, and $E_{s}$ is the energy function defined according to the type of $D_{s}$ (global or local dictionary). For convenience, the symbols used in this paper are listed in Table I.

\section{B. Sparse Representation and Dictionary Learning}

Sparse coding [31], [32] is a technique of finding a sparse representation for a signal with a small number of nonzero or significant coefficients corresponding to the atoms in a dictionary. To construct a dictionary $D_{s}$ to sparsely represent each patch $b_{s}^{k}$ 
TABLE I

NOTATION

\begin{tabular}{|c|c|}
\hline Symbols & Meanings \\
\hline$I$ & Input LR image \\
\hline$I^{d}$ & Down-scaled version of $I$ \\
\hline$I_{\mathrm{LF}}, I_{\mathrm{HF}}$ & Low-frequency and high-frequency parts of $I$ \\
\hline$I_{\mathrm{LF}}^{d}, I_{\mathrm{HF}}^{d}$ & Low-frequency and high-frequency parts of $I^{d}$ \\
\hline$x_{i}, y_{i}$ & Pair of the $i$-th corresponding HR and LR patches \\
\hline$x, y$ & HR and LR patch sets \\
\hline $\begin{array}{l}\left\{X^{B}, \mathcal{Y}^{B}\right\} \\
\left\{X^{N}, \mathcal{Y}^{N}\right\}\end{array}$ & Blocking and non-blocking HR/LR patch pairs \\
\hline$D_{B}, D_{N}$ & $\begin{array}{l}\text { Two sets of coupled dictionaries used for SR of blocking } \\
\text { and non-blocking patches }\end{array}$ \\
\hline$D_{N}^{\mathrm{HR}}, D_{N}^{\mathrm{LR}}$ & $\begin{array}{l}\text { Pair of dictionaries of corresponding HR/LR atoms for } \\
\text { non-blocking patches, forming } D_{N}\end{array}$ \\
\hline$D_{B}^{\mathrm{HR}}, D_{B}^{\mathrm{LR}}$ & $\begin{array}{l}\text { Pair of dictionaries of corresponding } \mathrm{HR} / \mathrm{LR} \text { atoms, } \\
\text { forming } D_{B}, \quad \text { where } D_{B}^{\mathrm{HR}}=\left[D_{B_{-} N}^{\mathrm{HR}} \mid D_{B_{-} B}^{\mathrm{HR}}\right] \text { and } \\
D_{B}^{\mathrm{LR}}=\left[D_{B_{-} N}^{\mathrm{LR}} \mid D_{B_{-} B}^{\mathrm{LR}}\right] .\end{array}$ \\
\hline$D_{B_{-} N}^{\mathrm{HR}}, D_{B_{-} N}^{\mathrm{LR}}$ & $\begin{array}{l}\text { Pair of non-blocking dictionaries consisting of } \\
\text { corresponding HR/LR atoms }\end{array}$ \\
\hline$D_{B_{-} B}^{\mathrm{HR}}, D_{B_{-} B}^{\mathrm{LR}}$ & $\begin{array}{l}\text { Pair of blocking dictionaries consisting of corresponding } \\
\text { HR/LR atoms }\end{array}$ \\
\hline$I_{\mathrm{HF}}^{N}, I_{\mathrm{HF}}^{B}$ & HR non-blocking and HR blocking components of $l_{\mathrm{HF}}$ \\
\hline $\begin{array}{l}b_{p}^{N}\left(b_{p}^{B}\right) \\
B_{p}^{N}\left(B_{p}^{B}\right)\end{array}$ & $\begin{array}{l}\text { The } p \text {-th non-blocking (blocking) patch in } I_{H F} \text { and its SR } \\
\text { result }\end{array}$ \\
\hline $\begin{array}{l}\theta_{p}^{N}\left(\theta_{p}^{B}\right) \\
\hat{\theta}_{p}^{N}\left(\hat{\theta}_{p}^{B}\right)\end{array}$ & $\begin{array}{l}\text { Sparse representation of } b_{p}^{N}\left(b_{p}^{B}\right) \text { and reconstructed sparse } \\
\text { representation of } b_{p} \text {, used to estimate } B_{p}^{N}\left(B_{p}^{B}\right)\end{array}$ \\
\hline$\hat{\theta}_{p_{-} N}^{B}$ & $\begin{array}{l}\text { Sparse representation of } b_{p}^{B} \text { with the coefficients } \\
\text { corresponding to the atoms in } D_{B B}^{\mathrm{LR}} \text {, being set to zeros. }\end{array}$ \\
\hline
\end{tabular}

extracted from component $I_{s}$ of image $I$, we may use a set of available training exemplars $y^{k}, k=1,2, \ldots, P$, to learn dictionary $D_{s}$ sparsifying $y^{k}$ by solving the following optimization problem:

$$
\min _{D_{s}, \theta^{k}} \sum_{k=1}^{P}\left(\frac{1}{2}\left\|y^{k}-D_{s} \theta^{k}\right\|_{2}^{2}+\lambda\left\|\theta^{k}\right\|_{1}\right)
$$

where $\theta^{k}$ denotes the sparse coefficient vector of $y^{k}$ with respect to $D_{s}$ and $\lambda$ is a regularization parameter.

\section{Proposed Joint SR And Deblocking Framework}

In this section, we first explain the proposed framework for self-learning-based SR. Then, in Section III-E, we will show how to extend the proposed framework to non-self-learningbased SR schemes. Fig. 3 depicts the proposed framework for self-learning-based joint SR and deblocking for enhancing a downscaled and highly compressed image. Our method is to formulate the image enhancement problem as an MCA-based image decomposition problem via sparse representation.

As illustrated in Fig. 3, an input LR image $I$ with blocking artifacts [Fig. 3(a)] and its downscaled version $I^{d}$ [Fig. 3(b)] are first roughly decomposed into their corresponding low-frequency (LF) parts, $I_{\mathrm{LF}}$ and $I_{\mathrm{LF}}^{d}$, and high-frequency (HF) parts, $I_{\mathrm{HF}}$ and $I_{\mathrm{HF}}^{d}$, respectively, via a filter. As a result, the respective essential visual information will be retained in the LF parts [Figs. 3(c) and 3(d)], while the blocking artifacts and edge/texture details will be included in the HF parts [see Figs. 3(e) and 3(f)] of the images. Then, we classify all patches in $I_{\mathrm{HF}}$ together with their corresponding patches in $I_{\mathrm{HF}}^{d}$ into two clusters: the "blocking" and "non-blocking" HR/LR patch pairs, respectively. Based on the two training sets of patch pairs extracted from the input image itself, we learn two sets of coupled dictionaries, $D_{B}$ and $D_{N}$, used for the SR of blocking and non-blocking patches, respectively, as illustrated in Figs. 3(g)-Figs. 3(i).

To achieve the SR of $I_{\mathrm{HF}}$, we perform patch-wise sparse reconstruction with the coupled dictionary set $D_{N}$ [Fig. 3(i)] for each patch without blocking artifacts in $I_{\mathrm{HF}}$. For each patch with blocking artifacts in $I_{\mathrm{HF}}$, we perform SR reconstruction based on $D_{B}$ (which consisits of two sub-dictionaries of HR and LR atoms, respectively), and MCA-based image decomposition to obtain the deblocked HR patch. As a result, the HF part $I_{\mathrm{HF}}$ can be simultaneously upscaled and decomposed into HR non-blocking and HR blocking components [Figs. 3(j) and $3(\mathrm{k})]$. We then add the HR non-blocking component of $I_{\mathrm{HF}}$ to the bicubic-interpolated $I_{\mathrm{LF}}$ [Fig. 3(1)] to obtain the final SR result of $I$, as illustrated in Fig. 3(m). The detailed method will be elaborated below.

\section{A. Preprocessing and Problem Formulation}

Without the need of pre-collecting enormous additional training patches for SR (e.g., in [9], [10], collection of additional training patches is required), the proposed method intends to extract training patches from an input LR image itself. Moreover, to achieve joint SR and deblocking, we convert the problem into the high-frequency domain of the input image and conduct the following preprocessing tasks. To model the relationship between LR and HR image patches, for an input LR image $I$ with blocking artifacts, we first down-scale $I$ to obtain its down-scaled version $I^{d}$, and then apply the BM3D (block-matching and 3D filtering) algorithm [33] to decompose $I$ into the LF part $\left(I_{\mathrm{LF}}\right)$ and $\mathrm{HF}$ part $\left(I_{\mathrm{HF}}\right)$, and decompose $I^{d}$ into $I_{\mathrm{LF}}^{d}$ and $I_{\mathrm{HF}}^{d}$, that is $I=I_{\mathrm{LF}}+I_{\mathrm{HF}}$, and $I^{d}=I_{\mathrm{LF}}^{d}+I_{\mathrm{HF}}^{d}$. Then, we identify two sets of HR/LR patch pairs as the training samples for learning the dictionaries used for SR and deblocking, where we extract each patch $\left(x_{i} \in \mathcal{X}\right)$ in the higher scale $\left(I_{\mathrm{HF}}\right)$ and its corresponding patch $\left(y_{i} \in \mathcal{Y}\right)$ in the lower scale $\left(I_{\mathrm{HF}}^{d}\right)$ with a certain magnification factor to form a coupled training patch pair. Then, we perform blocking artifact detection [20] on the HR part of each coupled patch pair (i.e., a patch from $I_{\mathrm{HF}}$ ) and classify all coupled patch pairs into two sets of "blocking" and "non-blocking" patch pairs: $\left\{\mathcal{X}^{B}, \mathcal{Y}^{B}\right\}$ and $\left\{\mathcal{X}^{N}, \mathcal{Y}^{N}\right\}$.

Based on the two sets of training samples, we propose to learn two sets of dictionaries, respectively, for SR of non-blocking patches and joint SR and deblocking of blocking patches, as detailed in Section III-B and Section III-C. Then, we formulate the SR of each input LR non-blocking patch $b_{p}^{N}$ (in $I_{\mathrm{HF}}$ ) as the following sparse representation problem:

$$
\hat{\theta}_{p}^{N}=\arg \min _{\theta_{p}^{N}}\left\|b_{p}^{N}-D_{N}^{\mathrm{LR}} \theta_{p}^{N}\right\|_{2}^{2}+\lambda\left\|\theta_{p}^{N}\right\|_{1}
$$

where $D_{N}^{\mathrm{LR}}$ denotes the learned LR dictionary of non-blocking atoms, $\theta_{p}^{N}$ is the sparse presentation of $b_{p}^{N}$ with respect to $D_{N}^{\mathrm{LR}}$, $\hat{\theta}_{p}^{N}$ is the solution of $\theta_{p}^{N}$ for minimizing (3), and $\lambda$ is a parameter controlling the sparsity penalty and representation fidelity. As a result, the SR result $B_{p}^{N}$ of $b_{p}^{N}$ can be obtained as follows:

$$
B_{p}^{N}=D_{N}^{\mathrm{HR}} \hat{\theta}_{p}^{N}
$$




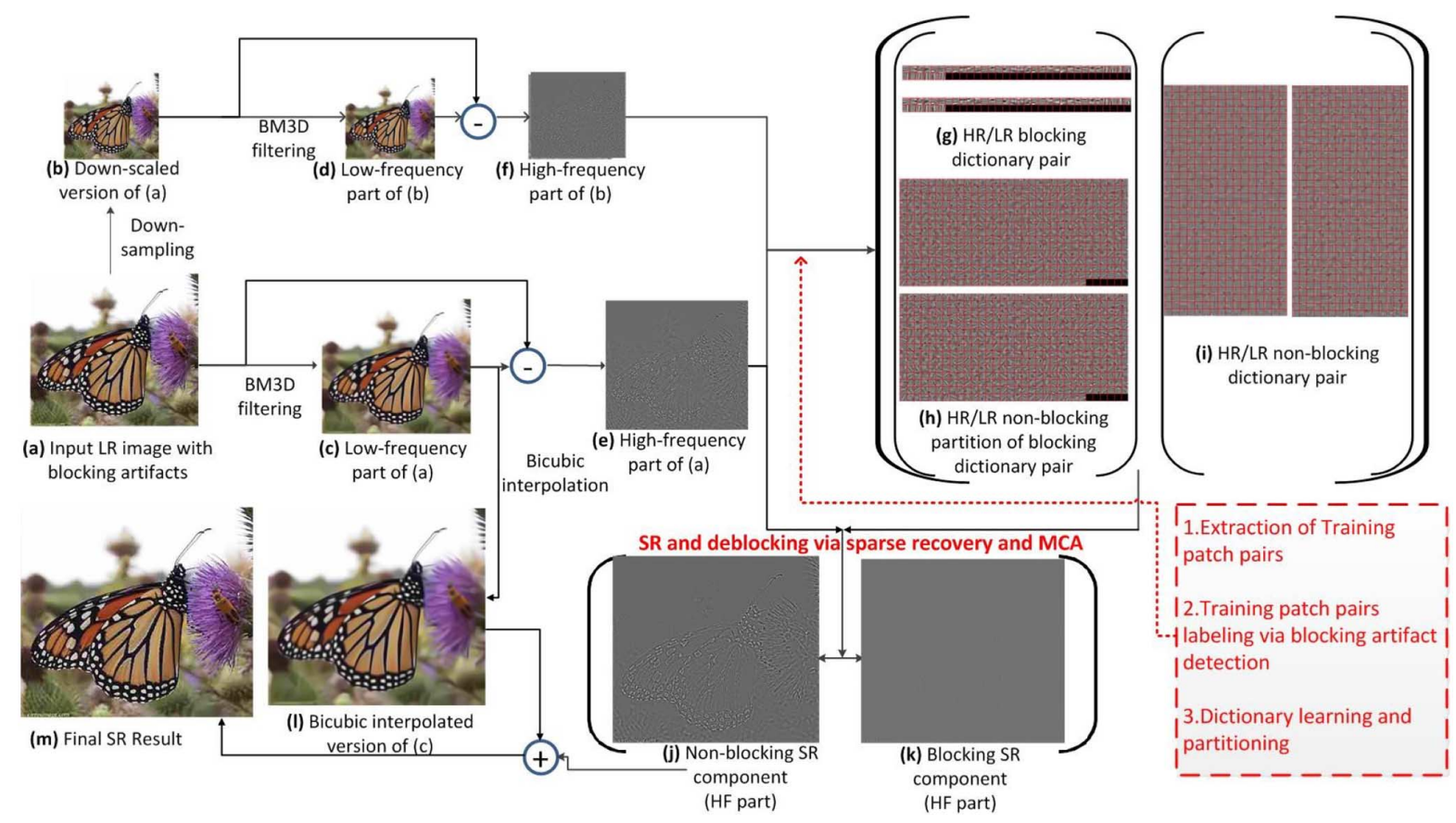

Fig. 3. Flowchart of the proposed self-learning-based super-resolution framework for a highly compressed image.

where $D_{N}^{\mathrm{HR}}$ is the learned HR dictionary of non-blocking atoms, which leads to the same sparse representation $\hat{\theta}_{p}^{N}$ for an HR patch as that for its corresponding LR patch with respect to $D_{N}^{\mathrm{LR}}$.

Moreover, we formulate the joint SR and deblocking for each input LR blocking patch $b_{p}^{B}$ (in $I_{\mathrm{HF}}$ ) as an MCA-based image decomposition problem via sparse representation

$$
\hat{\theta}_{p}^{B}=\arg \min _{\theta_{p}^{B}}\left\|b_{p}^{B}-D_{B}^{\mathrm{LR}} \theta_{p}^{B}\right\|_{2}^{2}+\lambda\left\|\theta_{p}^{B}\right\|_{1}
$$

where the definitions of $\theta_{p}^{B}$ and $\hat{\theta}_{p}^{B}$ are similar to those in (3), and $D_{B}^{\mathrm{LR}}=\left[D_{B_{-} N}^{\mathrm{LR}} \mid D_{B_{-} B}^{\mathrm{LR}}\right]$ is the learned LR dictionary based on the training samples with blocking artifacts, which can be further partitioned into $D_{B_{-} N}^{\mathrm{LR}}$ and $D_{B_{-} B}^{\mathrm{LR}}$, consisting of the nonblocking and blocking atoms, respectively. As a result, the joint SR and deblocking result $B_{p}^{B}$ of $b_{p}^{B}$ can be obtained by

$$
B_{p}^{B}=D_{B_{-} N}^{\mathrm{HR}} \hat{\theta}_{p_{-} N}^{B}
$$

where $D_{B_{-} N}^{\mathrm{HR}}$ is obtained from the partition of the learned HR dictionary $D_{B}^{\mathrm{HR}}$ based on the training samples with blocking artifacts, which can be further partitioned into $D_{B_{-} N}^{\mathrm{HR}}$ and $D_{B_{-} B}^{\mathrm{HR}}$, including the non-blocking and blocking atoms, respectively, and $\hat{\theta}_{p_{-}}^{B}$ is the sparse representation of $b_{p}^{B}$ obtained by solving (5) with the coefficients corresponding to the atoms in $D_{B_{-} B}^{\mathrm{LR}}$, being set to zero.

\section{B. Dictionary Learning for Single Image SR}

Based on the extracted HR/LR training patch pairs without blocking artifacts $\left(\left\{\mathcal{X}^{N}, \mathcal{Y}^{N}\right\}\right)$ from $I_{\mathrm{HF}}$ itself, we intend to learn a couple of dictionaries $\left(D_{N}^{\mathrm{HR}}\right.$ and $\left.D_{N}^{\mathrm{LR}}\right)$ to model the relationships between HR and LR image patches. Similar to the coupled dictionary training method proposed in [10], we treat the set $\mathcal{Y}^{N}$ of LR training patches as the observation space, and the set $\mathcal{X}^{N}$ of HR training patches as the latent space, where the patches have sparse representations with respect to certain dictionaries. Patches (LR) in $\mathcal{Y}^{N}$ are observations, while patches (HR) in $\mathcal{X}^{N}$ are what we want to recover. The problem is to find a coupled dictionary pair $D_{N}^{\mathrm{HR}}$ and $D_{N}^{\mathrm{LR}}$ for spaces $\mathcal{X}^{N}$ and $\mathcal{Y}^{N}$, respectively, such that given an input LR patch $y_{i} \in \mathcal{Y}^{N}$, we can use its sparse representation with respect to $D_{N}^{\mathrm{LR}}$ to reconstruct the corresponding latent HR patch $x_{i} \in \mathcal{X}^{N}$ with respect to $D_{N}^{\mathrm{HR}}$. Hence, for each coupled patch pair $\left\{x_{i}, y_{i}\right\}$, a desired pair of coupled dictionaries $D_{N}^{\mathrm{HR}}$ and $D_{N}^{\mathrm{LR}}$ should satisfy

$$
\begin{aligned}
& \hat{\theta}_{i}=\arg \min _{\theta_{i}}\left\|y_{i}-D_{N}^{\mathrm{LR}} \theta_{i}\right\|_{2}^{2}+\lambda\left\|\theta_{i}\right\|_{1}, \\
& \hat{\theta}_{i}=\arg \min _{\theta_{i}}\left\|x_{i}-D_{N}^{\mathrm{HR}} \theta_{i}\right\|_{2}^{2}
\end{aligned}
$$

where $\hat{\theta}_{i}$ is the sparse representation of $y_{i}$ with respect to $D_{N}^{\mathrm{LR}}$, and also the sparse representation of $x_{i}$ for recovering $x_{i}$ with respect to $D_{N}^{\mathrm{HR}}$.

Given input $y_{i}$, to learn a coupled dictionary pair $D_{N}^{\mathrm{HR}}$ and $D_{N}^{\mathrm{LR}}$ for reconstructing $x_{i}$ with respect to $D_{N}^{\mathrm{HR}}$, the problem can be formulated as [10]

$$
\min _{D_{N}^{\mathrm{HR}}, D_{N}^{\mathrm{LR}}} \frac{1}{P} \sum_{i=1}^{P} L\left(D_{N}^{\mathrm{HR}}, D_{N}^{\mathrm{LR}}, x_{i}, y_{i}\right)
$$

s.t. $\hat{\theta}_{i}=\arg \min _{\theta_{i}}\left\|y_{i}-D_{N}^{\mathrm{LR}} \theta_{i}\right\|_{2}^{2}+\lambda\left\|\theta_{i}\right\|_{1}, i=1,2, \cdots, P$, where $P$ is the number of training patch pairs, and $L(\cdot)$ represents a cost function for ensuring a good representation of $y_{i}$ with respect to $D_{N}^{\mathrm{LR}}$, and minimizing the reconstruction error of $x_{i}$ with respect to $D_{N}^{\mathrm{HR}}$, defined as follows:

$$
L=\frac{1}{2}\left[\alpha\left\|D_{N}^{\mathrm{HR}} \hat{\theta}_{i}-x_{i}\right\|_{2}^{2}+(1-\alpha)\left\|D_{N}^{\mathrm{LR}} \hat{\theta}_{i}-y_{i}\right\|_{2}^{2}\right]
$$


where $\alpha(0<\alpha<1)$ is used to balance the two reconstruction errors. The objective function in (9) can be minimized by alternatively optimizing over $D_{N}^{\mathrm{HR}}$ and $D_{N}^{\mathrm{LR}}$, while keeping the other fixed. More details about the coupled dictionary learning can be found in [10]. The main difference between the proposed method and the approach proposed in [10] is that all of the training patches are extracted from an input LR image itself in our approach while enormous additional training patches are required in [10].

\section{Dictionary Learning for Image Decomposition}

For the extracted HR/LR training patch pairs with blocking artifacts, we need to not only learn a coupled dictionary pair $D_{B}^{\mathrm{HR}}$ and $D_{B}^{\mathrm{LR}}$ for SR purpose, but also identify the "blocking/non-blocking atoms" in the two dictionaries for achieving MCA-based deblocking. Similar to the employed coupled dictionary learning technique described in Section III-B, we first learn $D_{B}^{\mathrm{HR}}$ and $D_{B}^{\mathrm{LR}}$ by solving (9), where the parameters are replaced accordingly. Then, we analyze the atoms constituting $D_{B}^{\mathrm{HR}}$, and find that these atoms can be roughly divided into two clusters (sub-dictionaries) for representing the non-blocking and blocking components of $I_{\mathrm{HF}}$, respectively. That is, even if the training patches extracted from $I_{\mathrm{HF}}$ are classified into blocking patches based on [20], two different kinds of atoms in terms of blocking and non-blocking artifacts can still be analyzed after the dictionary learning process.

Based on our previous work in [22], we modify the HOG (histograms of oriented gradients) descriptor [34] to describe each atom in $D_{B}^{\mathrm{HR}}$ (the HOG features of the atoms in the dictionary learned from HR patches should be more significant than those from LR patches). Since blocking artifacts are visually noticeable changes in pixel values along block boundaries, it is only required to consider the horizontal and vertical gradients in each dictionary atom. Hence, we modify the original $\mathrm{HOG}$ to calculate only the histogram over the intervals of angles around $0^{\circ}, 180^{\circ}, 90^{\circ}$, and $270^{\circ}$. After extracting the HOG feature for each atom in $D_{B}^{\mathrm{HR}}$, we first apply the $K$-means algorithm to classify all of the atoms in $D_{B}^{\mathrm{HR}}$ into two clusters, $D_{1}$ and $D_{2}$, based on their horizontal HOG feature descriptors. Then, we calculate the variance of gradient directions for each atom $d_{i j}$ in cluster $D_{i}$, as $V G_{i j}$, $i=1,2$. We subsequently calculate the mean of $V G_{i j}$ for each cluster $D_{i}$ as $M V G_{i}$. Based on the fact that the edge directions of the samples of an atom with horizontal (or vertical) blocking artifacts should be consistent, i.e., the variance of gradient directions for a "horizontal (or vertical) blocking" atom should be generally smaller than those of the other atoms with no remarkably dominating edge direction, we identify the cluster with the smaller $M V G_{i}$ as horizontal blocking sub-dictionary. Then, similarly the other cluster can be further classified to obtain the "vertical blocking" sub-dictionary and non-blocking sub-dictionary $D_{B-N}^{\mathrm{HR}}$. In addition, the identified horizontal and vertical blocking sub-dictionaries constitute the blocking sub-dictionary $D_{B}^{\mathrm{HR}}$. That is, $D_{B}^{\mathrm{HR}}=\left[D_{B_{-} N}^{\mathrm{HR}} \mid D_{B_{-} B}^{\mathrm{HR}}\right]$. Meanwhile, the atoms in $D_{B}^{\mathrm{LR}}$ can also be classified into two clusters according to their corresponding atoms in $D_{B}^{\mathrm{HR}}$ to form the blocking and non-blocking dictionaries, $D_{B_{-} N}^{\mathrm{LR}}$ and $D_{B_{-} B}^{\mathrm{LR}}$,

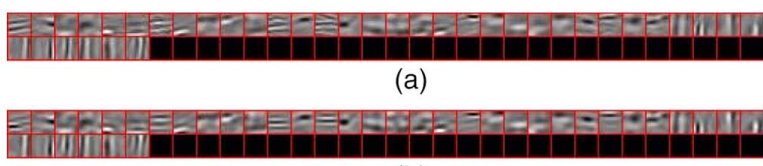

(b)

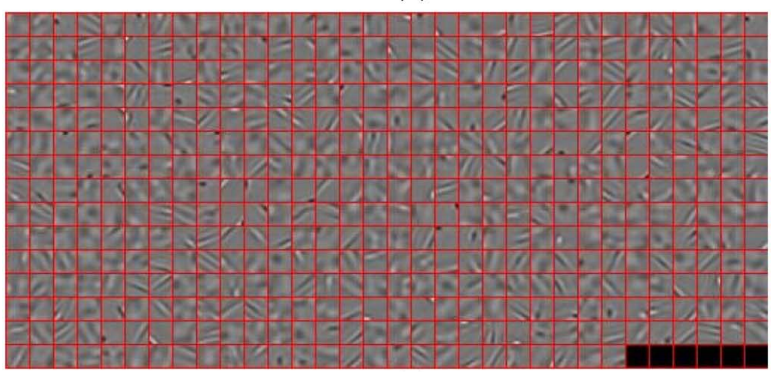

(c)

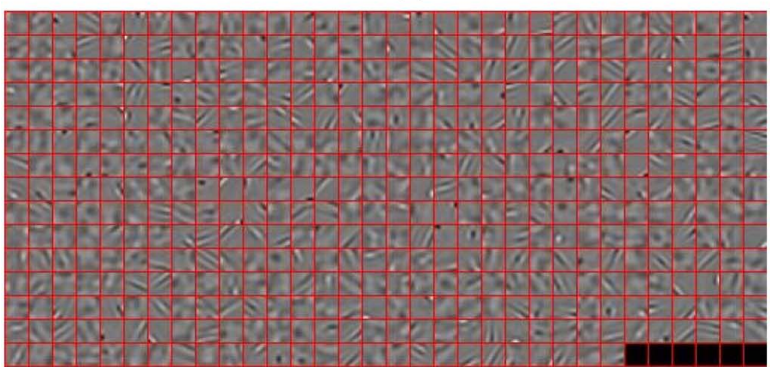

(d)

Fig. 4. Highlight of the two learned dictionary pairs based on the extracted HR/LR training patch pairs with blocking artifacts from Fig. 3(a): (a) the dictionary of HR atoms with blocking artifacts $\left(D_{B-B}^{\mathrm{HR}}\right)$; (b) the dictionary of LR atoms with blocking artifacts $\left(D_{B-B}^{\mathrm{LR}}\right) ;($ c) the dictionary of HR atoms without blocking artifacts $\left(D_{B-N}^{\mathrm{HR}}\right)$, i.e., non-blocking partition of $D_{B}^{\mathrm{HR}}$; and (d) the dictionary of LR atoms without blocking artifacts $\left(D_{B}^{\mathrm{LR}}\right)$, non-blocking partition of $D_{B}^{\mathrm{LR}}$.

of LR atoms, i.e., $D_{B}^{\mathrm{LR}}=\left[D_{B_{-} N}^{\mathrm{LR}} \mid D_{B_{-} B}^{\mathrm{LR}}\right]$. As an example highlighting Figs. 3(g) and Fig. 3(h), Fig. 4 shows the two learned dictionary pairs based on the extracted HR/LR training patch pairs with blocking artifacts from Fig. 3(a). To summarize the training patch extraction and dictionary learning processes in our framework, the red dotted line region in Fig. 3 is highlighted and illustrated in Fig. 5.

\section{Image SR and Deblocking via Sparse Reconstruction}

After learning the six dictionaries, $D_{N}^{\mathrm{HR}}, D_{N}^{\mathrm{LR}}, D_{B_{-} N}^{\mathrm{HR}}$, $D_{B_{-} B}^{\mathrm{HR}}, D_{B_{-} N}^{\mathrm{LR}}$, and $D_{B_{-} B}^{\mathrm{LR}}$, based on the training patches extracted from $I_{\mathrm{HF}}$, joint SR and deblocking of $I_{\mathrm{HF}}$ can be efficiently achieved via patch-wise sparse recovery as follows.

1) SR for non-blocking patches: In this case, only SR is required to be performed for each patch. For each input LR non-blocking patch $b_{p}^{N}$ in $I_{\mathrm{HF}}$, we first calculate its sparse representation $\hat{\theta}_{p}^{N}$ with respect to $D_{N}^{\mathrm{LR}}$ via (3). Based on the employed coupled dictionary learning algorithm described in Section III-B, the spare representation can also be used to recover its HR version $B_{p}^{N}$ with respect to $D_{N}^{\mathrm{HR}}$ via (4).

2) Joint SR and deblocking for blocking patches: In this case, both SR and deblocking are jointly performed for each patch, For each input LR blocking patch $b_{p}^{B}$ in $I_{\mathrm{HF}}$, we first perform MCA-based image decomposition via sparse representation with respect to $D_{B}^{\mathrm{LR}}=\left[D_{B_{-} N}^{\mathrm{LR}} \mid D_{B_{-} B}^{\mathrm{LR}}\right]$ to 


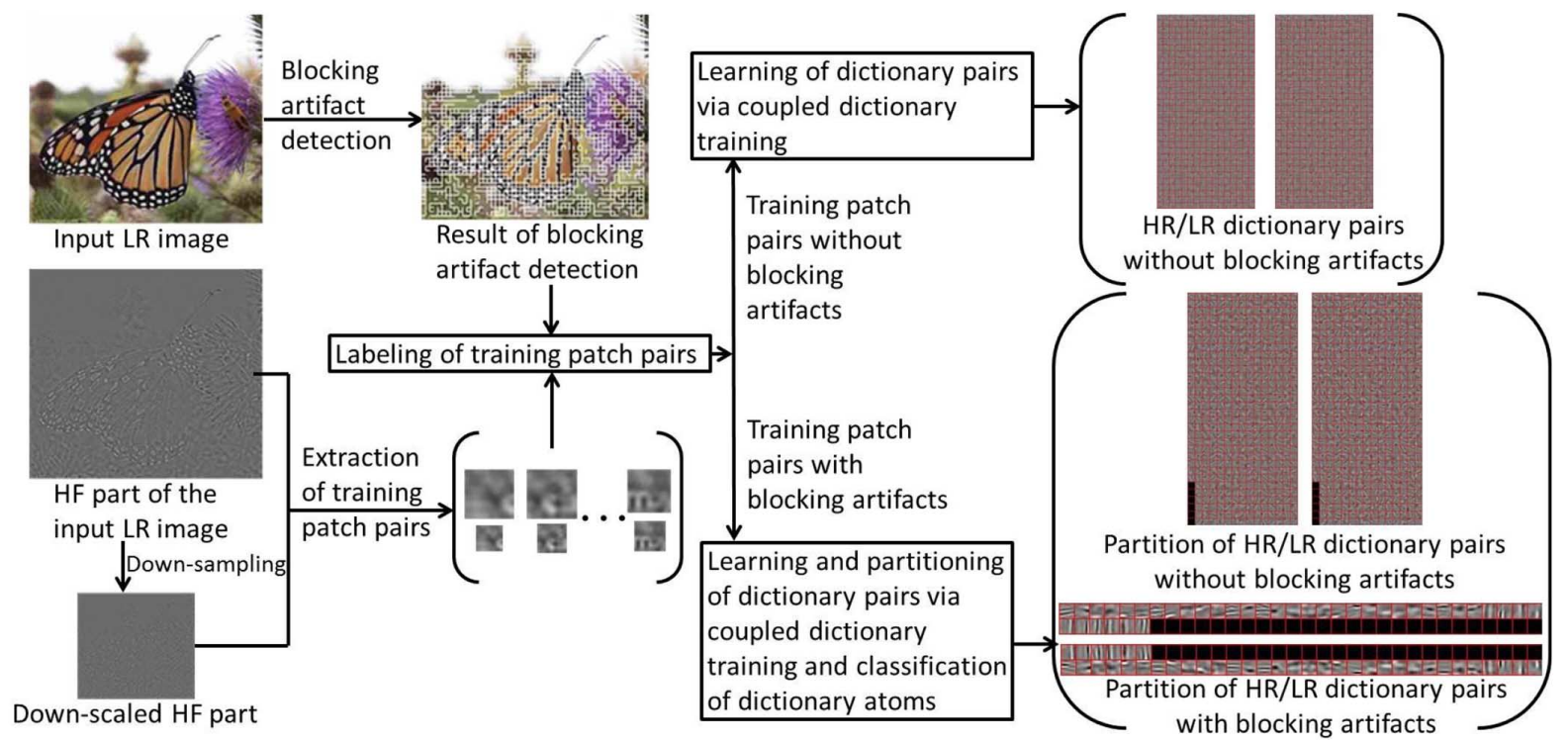

Fig. 5. Illustration of the proposed training patch extraction and dictionary learning processes in the proposed framework (highlight and illustration of the red dotted line region in Fig. 3).

find the atoms which actually contribute for representing the non-blocking part of $b_{p}^{B}$ via (5). We then set the coefficients corresponding to the atoms in $D_{B-B}^{\mathrm{LR}}$ in $\hat{\theta}_{p}^{B}$ (the solved sparse representation of $b_{p}^{B}$ ) to zero to obtain $\hat{\theta}_{p_{-} N}^{B}$ (the estimated sparse representation of the non-blocking component of $b_{p}^{B}$ ). As a result, the joint $\mathrm{SR}$ and deblocking of $b_{p}^{B}$ can be achieved by using the sparse representation $\hat{\theta}_{p_{-} N}^{B}$ with respect to $D_{B_{-} N}^{\mathrm{HR}}$ via (6).

Then, the recovered and deblocked HR patches are tiled together to reconstruct the HR version $I_{\mathrm{HF}}^{N}$ of $I_{\mathrm{HF}}$, where the average of multiple reconstructs is taken for each pixel in the overlapping region as its final recovery. Finally, we add the HR details $I_{\mathrm{HF}}^{N}$ to the bicubic-interpolated $I_{\mathrm{LF}}$ to obtain the final SR result of $I$. To briefly summarize the proposed framework and the relationship between it and the six learned dictionaries, the proposed joint SR and deblocking algorithm is summarized in Table II.

\section{E. Extension to Non-Self-Learning-Based SR}

Besides the self-learning-based scheme mentioned above, the proposed framework can also be easily integrated into other non-self-learning SR methods as illustrated in Fig. 6. Note that the main difference is that the dictionary used in self-learning $\mathrm{SR}$ is learned from the input LR image itself, whereas the non-self-learning SR algorithms (e.g., the sparse-coding SR in [9]) needs to learn a dictionary from a pre-collected training image set. In the extension shown in Fig. 6, each HR image in the training set is first downscaled and compressed to obtain its LR version with blocking artifacts, which are then localized by the following blocking artifact detection, so as to form a HR/LR training pair. Subsequently, the training patch extraction and dictionary learning processes described in Fig. 5 and Section III-C are performed on the blocking and non-blocking
TABLE II

Proposed JoINT SR AND DeBlocking ALgORITHM BASED ON SIX LEARNED DiCTIONARIES

Input: a LR image $I$ with blocking artifacts.

Output: the blocking artifacts-removed HR version of $I$.

\section{Learning phase}

1. Extraction of training HR/LR patch pairs from the HF part $\left(I_{\mathrm{HF}}\right)$ of $I$.

2. Classification of the training patches into training patch pairs $\left(\left\{X^{N}, Y^{N}\right\}\right)$ without blocking artifacts and training patch pairs $\left(\left\{X^{B}, \mathcal{Y}^{B}\right\}\right)$ with blocking artifacts.

3. Learning of HR/LR dictionary pairs $\left(D_{N}^{\mathrm{HR}}\right.$ and $\left.D_{N}^{\mathrm{LR}}\right)$ without blocking artifacts via coupled dictionary training based on $\left\{X^{N}, y^{N}\right\}$.

4. Learning of HR/LR dictionary pairs $\left(D_{B}^{\mathrm{HR}}\right.$ and $\left.D_{B}^{\mathrm{LR}}\right)$ with blocking artifacts via coupled dictionary training based on $\left\{X^{B}, \mathcal{Y}^{B}\right\}$.

5. Partitioning $D_{B}^{\mathrm{HR}}$ and $D_{B}^{\mathrm{LR}}$ into $D_{B_{-} N}^{\mathrm{HR}}$ and $D_{B_{-} B}^{\mathrm{HR}}$, and $D_{B_{-} N}^{\mathrm{LR}}$ and $D_{B_{-} B}^{\mathrm{LR}}$, respectively, to obtain $\mathrm{HR} / \mathrm{LR}$ dictionary pairs $\left(D_{B_{-} N}^{\mathrm{HR}}\right.$ and $\left.D_{B_{-} N}^{\mathrm{LR}}\right)$ without blocking artifacts, and $\mathrm{HR} / \mathrm{LR}$ dictionary pairs $\left(D_{B_{-} B}^{\mathrm{HR}}\right.$ and $\left.D_{B_{-} B}^{\mathrm{LR}}\right)$ with blocking artifacts, where $D_{B_{-} B}^{\mathrm{HR}}$ can be discarded.

Reconstruction phase

1. For each LR patch without blocking artifacts of $I_{\mathrm{HF}}$, perform sparse decomposition with $D_{N}^{\mathrm{LR}}$ via (3) and reconstruct its $\mathrm{HR}$ version with $D_{N}^{\mathrm{HR}}$ via (4).

2. For each LR patch with blocking artifacts of $I_{\mathrm{HF}}$, perform sparse decomposition with $D_{B}^{\mathrm{LR}}=\left[D_{B_{-} N}^{\mathrm{LR}} \mid D_{B_{-} B}^{\mathrm{LR}}\right]$ via (5) and keep only the sparse coefficients corresponding to $D_{B_{-} N}^{\mathrm{LR}}$. Then, reconstruct its blocking artifacts-removed $\mathrm{HR}$ version with $D_{B_{-}}^{\mathrm{HR}}$ via (6).

3. Return the integration of the reconstructed blocking artifacts-removed HR version of $I_{\mathrm{HF}}$ and the bicubic-interpolated LF part $\left(I_{\mathrm{LF}}\right)$ of $I$.

patch pairs extracted from the training HR/LR image pairs to learn and partition the blocking and non-blocking HR/LR sub-dictionaries. Consequently, based on the learned sub-dictionaries, joint SR and deblocking can be achieved by the proposed image decomposition method described in Fig. 3 and Section III-D. Based on the method shown in Fig. 6, we integrate our framework with the sparse-coding SR (SCSR) in [9], which is a state-of-the-art non-self-learning SR scheme, and will report the results in the following section. 


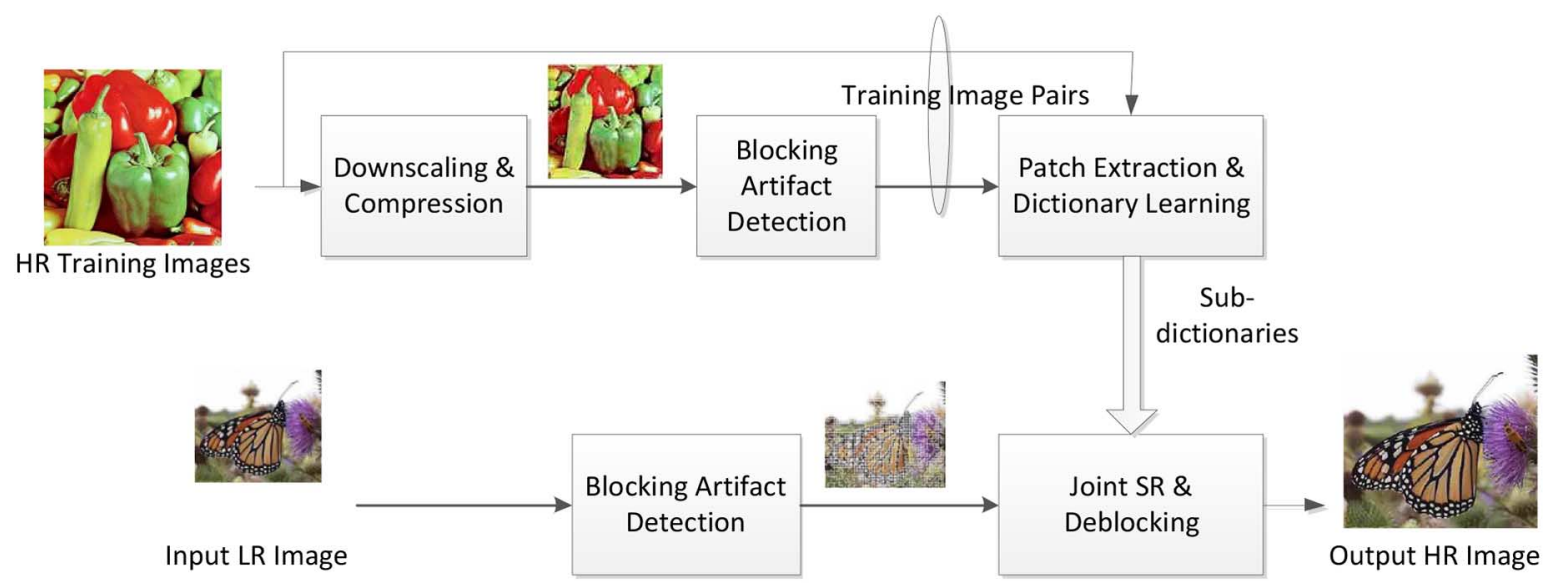

Fig. 6. Extension of the proposed framework to other non-self-learning-based SR methods.

\section{EXPERIMENTAL RESULTS}

\section{A. Visual Quality Evaluation}

Several LR JPEG images and YouTube videos with blocking artifacts were used to evaluate the performance of the proposed method. All the test dataset and results can be downloaded from our project web site. ${ }^{2}$ In our experiments, all of the test LR images were compressed by JPEG with quality factor (QF) ranging from 15 to 25. Different from [15], where the JPEG compression $\mathrm{QF}$ is required to be known in advance, our approach does not require any prior knowledge (including QF) about an input LR image. The parameter settings of the proposed method are described as follows. For each test LR image of size ranging from $140 \times 140$ to $442 \times 400$, the upscaling factor, HR/LR patch sizes, the number of training iterations for dictionary learning, and the size (number of atoms) of each learned dictionary (including $D_{N}^{\mathrm{LR}}, D_{N}^{\mathrm{HR}}, D_{B}^{\mathrm{LR}}$, and $\left.D_{B}^{\mathrm{HR}}\right)$ are set to $2,16 \times 16 / 8 \times 8$, 100 , and 1024, respectively. Our framework learns two pairs of dictionaries, respectively, for training patches with and without blocking artifacts, where each dictionary pair contains 512 pairs of HR/LR atoms, resulting in the total dictionary size of 1024 . Besides, the regularization parameter $\lambda$ used in the dictionary learning step in (9) is empirically set to 0.15 , and the number of non-zero coefficients to be solved in each sparse reconstruction step in our method is set to 20 .

To highlight the advantage of the proposed joint self-learning SR and deblocking approach for a single image, we compare our method with four upscaling methods, including the bicubic interpolation [4], coupled dictionary training-based SR with extra training samples (denoted by coupled-training-based) [10], self-learning SR (denoted by SelfSR, similar to [14]), and a cascading approach including the BM3D deblocking method [34], followed by the SelfSR method (denoted by Cascading-based), where the latter three methods are all based on dictionary learning and their respective dictionary sizes are all set to 1024 for fair comparisons with our method. Note that based on our experiments for all of the compared methods, reducing the dictionary size to some extent (smaller than 1024)

\footnotetext{
2“NTHU Image/Video SR+Denoising Project," [Online]. Available: http:// www.ee.nthu.edu.tw/cwlin/SR_Denoising/index.html
}

will significantly degrade SR results, whereas increasing the dictionary size (larger than 1024) will increase the computational complexity without significant improvement on SR visual quality. Moreover, the number of non-zero coefficients to be solved in each sparse reconstruction step in the three compared methods is also set to 20. Here, the SelfSR method used for comparison is based on our framework without performing blocking artifact removal, which mainly relies on the dictionary learning based on HR/LR training patch pairs proposed in [9], [10] and the employed self-similarity characteristic of an input LR image proposed in [14].

Fig. 7 and Fig. 8 show some SR results obtained by the proposed method and the compared methods. The enlarged images in Fig. 7 and Fig. 8 illustrate that the visual qualities of our SR results are visually superior to the results obtained by the pure SR approaches, which show significantly magnified blocking artifacts. Moreover, our method also outperforms the cascading approach based on the fact that the application of deblocking prior to SR would inevitably lead to loss of some image details, which is usually hard to be recovered in the following SR stage.

We then further evaluate the performance of the proposed self-learning-based method on some real-world low-quality LR images/video frames with significant blocking artifacts, downloaded from the Internet (e.g., from YouTube ). These testing data might be downscaled by any down-sampling operations and highly compressed via any block-based compression algorithm. Fig. 9 shows some snapshots of the SR results obtained by the proposed method and the bicubic interpolation method [4] for a YouTube video. It can be similarly observed from Fig. 9 that the visual quality of our SR results is significantly better than the results obtained by the bicubic interpolation, especially for very low-quality image/video frames. More test results on some videos downloaded from YouTube can be found in our project web site, where all the source inks are provided. Due to the space limit, we do not show the results of the non-self-learning-based schemes.

We also conduct a subjective user study to evaluate the performances of various SR schemes (with/without deblocking) for 9 test images. In the subjective paired comparisons test [37], 20 subjects are shown with two side-by-side upscaled images obtained using different SR methods (in a random order) at a 


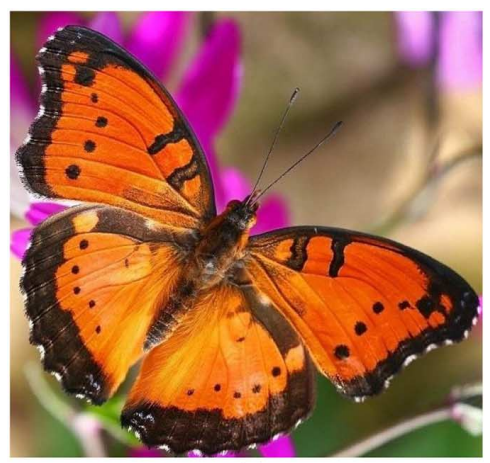

(a)

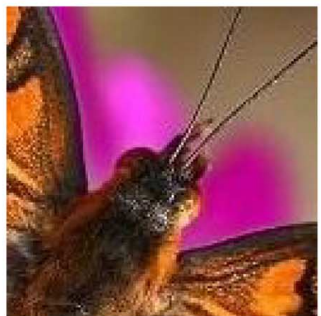

(d)

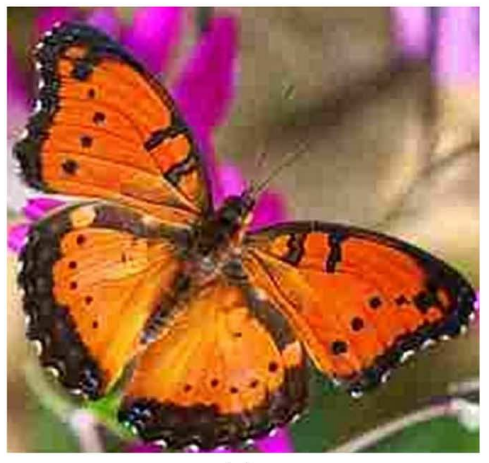

(g)

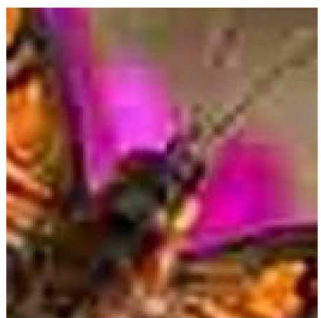

(j)

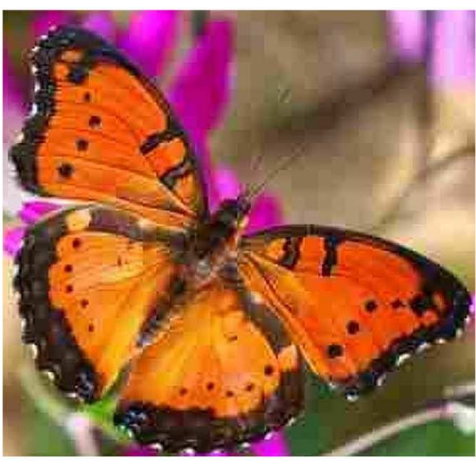

(b)

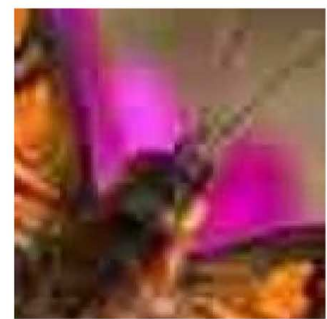

(e)

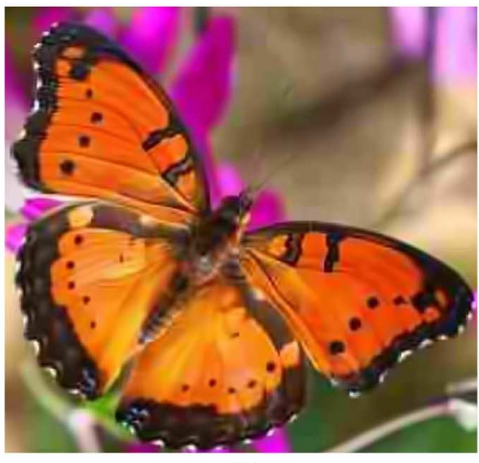

(h)

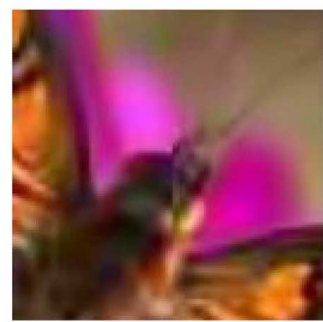

(k)

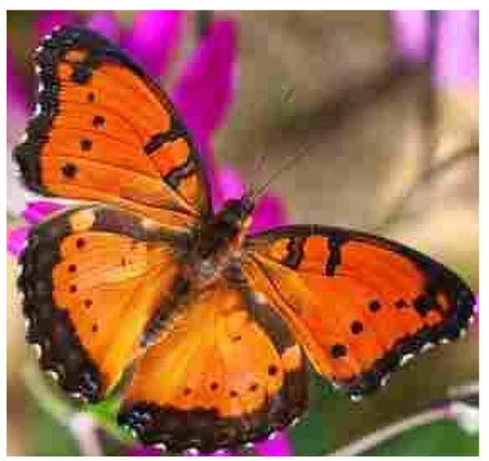

(c)

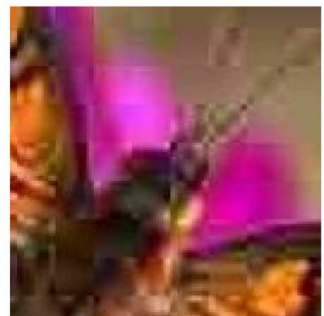

(f)

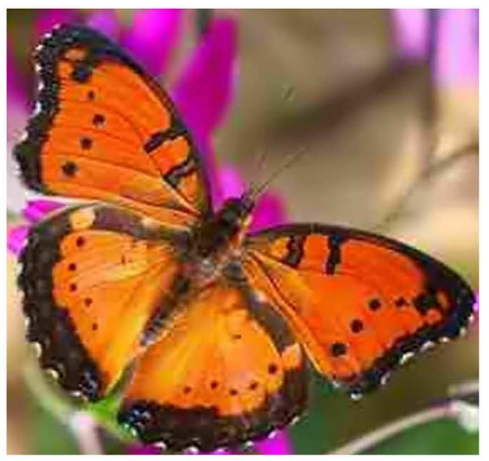

(i)

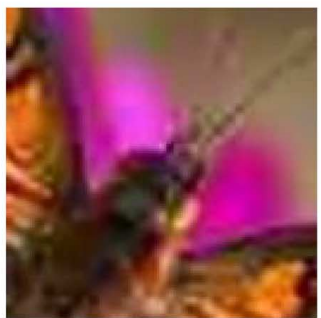

(I)

Fig. 7. SR results: (a) the original HR image; and the SR versions of the input LR image (LR and compressed version of (a), QF $=15)$ via the: (b) 7nbsp;bicubic [4]; (c) coupled-training-based [10]; (d) local highlight of (a); (e) local highlight of (b); (f) local highlight of (c); (g) SelfSR [14]; (h) cascading-based; (i) proposed method; (j) local highlight of (g); (k) local highlight of (h); and (l) local highlight of (i).

time, and are asked to choose their preference from the two compared images. The subjects include 15 males and 5 females, whose ages range from 25 to 30 . The test device is a full-HD 24-inch LCD display with color temperature $6500 \mathrm{~K}$. In the subjective test, we compare the proposed method with the original SR method [self-learning SR (SelfSR) or sparse-coding SR (SCSR)] without deblocking, Cascade type I (SR followed by deblocking), Cascade type II (deblocking followed by SR) for 9 test images. Each SR method is pairwisely compared with the others by totally $3 \times 9 \times 20=540$ times, meaning that 180 comparisons are made between two methods for the 9 test images. The ratio between the preference vote for one method and that for another method is used to compare the performances between the two methods. For example, if one method is preferred $80 \%$ of the time, the SR versions of all test images obtained by this method receive $80 \%$ of the preference vote, indicating that the method is visually preferred to the compared method in general.

Table III and Table IV show the winning frequency matrices [37] of the 20 subjects' preferences in subjective paired comparisons on the SR results obtained using the four different schemes based on the SelfSR and SCSR, respectively, where the numbers in each row indicate the times of the method preferred than the compared in the column. Both tables indicate that, compared 


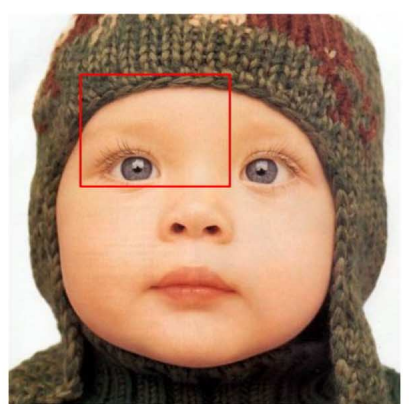

(a)

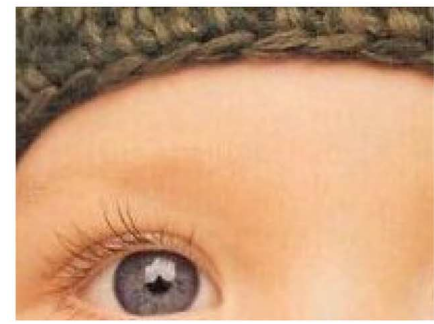

(d)

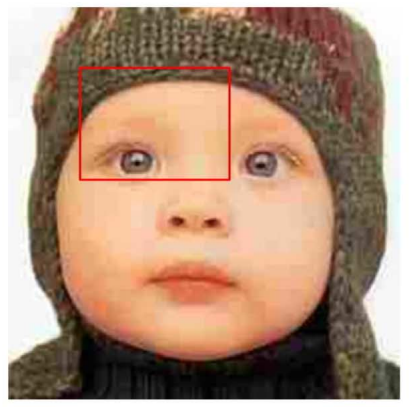

(g)

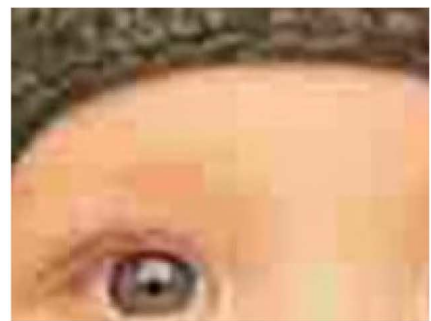

(j)

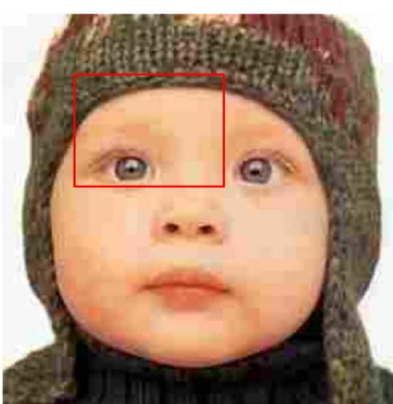

(b)

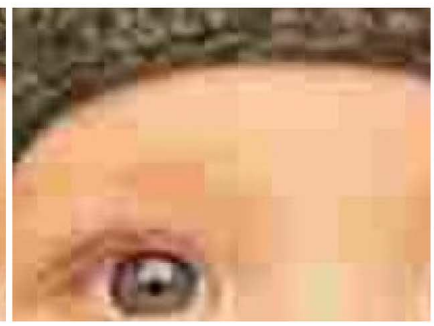

(e)

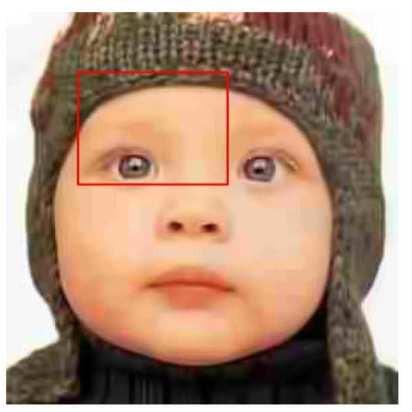

(h)

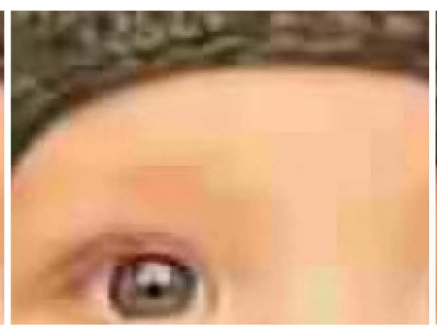

(k)

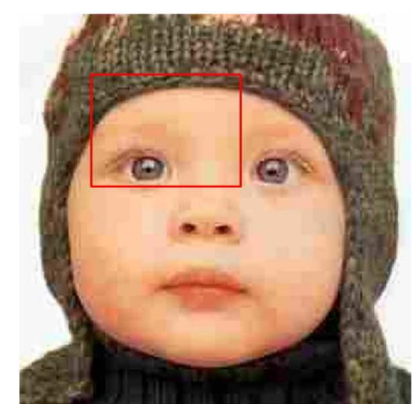

(c)

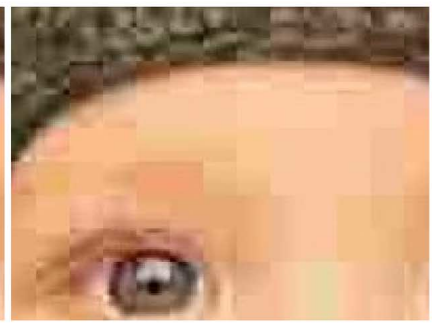

(f)

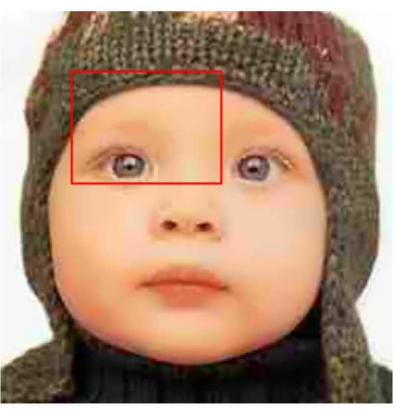

(i)

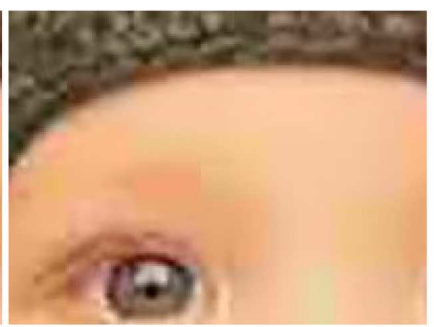

(I)

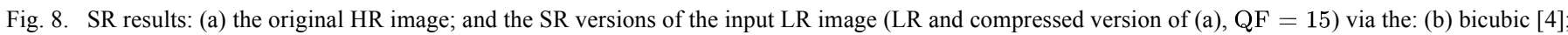

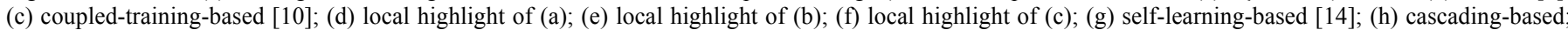
(i) proposed method; (j) local highlight of (g); (k) local highlight of (h); and (l) local highlight of (i).

to the other schemes, our method achieves better subjective visual quality in $78 \% \sim 88 \%$ and $80 \% \sim 92 \%$ of the paired comparisons based on the two different SR frameworks, respectively. Moreover, Table V lists the subjective paired comparison results of the SelfSR, SCSR, Proposed SelfSR (jointSelfSR + Deblocking), and Proposed SCSR (jointSCSR + deblocking) methods. the results shows that, the proposed SelfSR is preferred to the proposed SCSR subjectively in $64 \%$ of paired comparisons, and significantly outperforms the original SelfSR and SCSR methods in terms of subjective visual quality.

Based on our experimental results for individual test images, the performances of the proposed methods are dependent on the content characteristics (edges, textures, and smooth regions) of input LR image, summarized as follows.
1) Compared to SR without deblocking (i.e., the original SCSR and SelfSR), our methods are significantly more effective for images with strong edges, textures, and smoothing regions.

2) Compared to SR with deblocking, our methods achieve significant visual quality improvement for image regions with strong edges and textures. As for smooth regions, since blocking artifacts in such regions can be effectively mitigated using the deblocking part of the cascading-based approaches as well, our methods achieve comparable visual quality with the cascading-based approaches.

3) In general, our methods achieve overall visual quality improvement over existing SR methods with or without deblocking. 


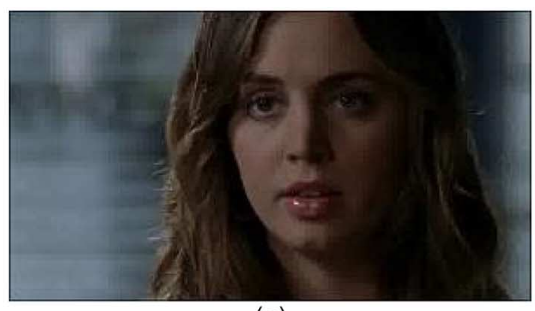

(a)

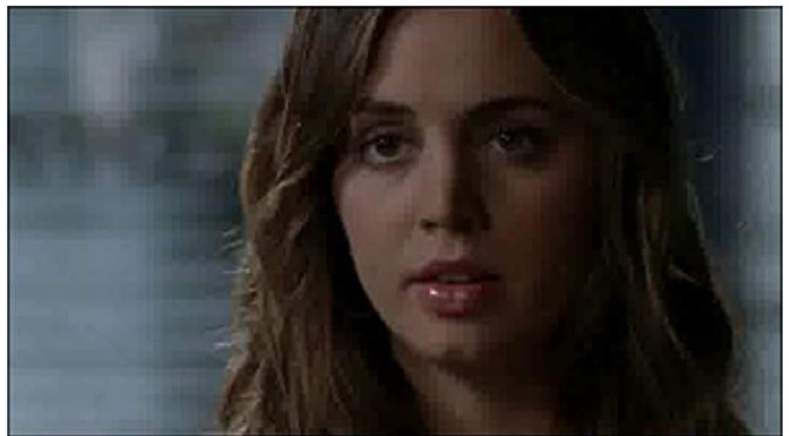

(b)

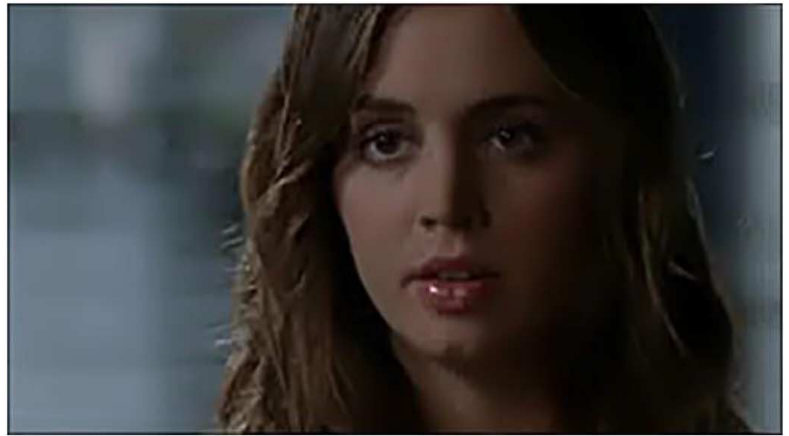

(c)

Fig. 9. SR results: (a) the original low-quality video frame obtained from the Internet; the SR versions of (a) via (b) bicubic [4]; and (c) the proposed method.

TABLE III

WinNing FreQuency MATRIX of SubJective PAIRED COMPARISONS BETWEen SELF-LEARNING SR (SELFSR), BM3 D + SelfSR, SelfSR + BM3 D AND THE PROPOSED JOINT SelfSR + Deblocking

\begin{tabular}{cccccc}
\hline & $\begin{array}{c}\text { Proposed } \\
\text { SelfSR }\end{array}$ & Cascade I & Cascade II & SelfSR & Average \\
\hline Proposed SelfSR & - & $\begin{array}{c}\mathbf{1 4 6} \\
\mathbf{( 8 1} \%)\end{array}$ & $\begin{array}{c}\mathbf{1 4 6} \\
\mathbf{( 8 1 \% )}\end{array}$ & $\begin{array}{c}\mathbf{1 6 8} \\
\mathbf{( 9 3 \% )}\end{array}$ & $\mathbf{8 5 . 2 \%}$ \\
\hline $\begin{array}{c}\text { Cascade I } \\
\text { (SelfSR+BM3D) }\end{array}$ & $\begin{array}{c}\mathbf{( 1 9 \% )} \\
\text { Cascade II }\end{array}$ & - & $\begin{array}{c}88 \\
(49 \%)\end{array}$ & $\begin{array}{c}139 \\
(77 \%)\end{array}$ & $48.3 \%$ \\
\hline (BM3D+SelfSR) & $(19 \%)$ & $\begin{array}{c}92 \\
(51 \%)\end{array}$ & - & $\begin{array}{c}137 \\
(76 \%)\end{array}$ & $48.7 \%$ \\
\hline SelfSR & 12 & 41 & 43 & - & $17.8 \%$ \\
\hline
\end{tabular}

\section{B. Limitations}

In our SR framework, we focus on performing SR for a highly compressed image while removing the visible blocking artifacts of the image. Nevertheless, the ringing artifact, not considered in this work, also usually occurs in a compressed image. Note, de-ringing can also be incorporated into the proposed MCA-based framework by using ringing artifact detection (e.g., [36]) to localize the ringing artifacts to learn the HOG characteristics of ringing artifacts and then use the
TABLE IV

WinNING FrEQUenCy MATRIX OF SUBJECTIVE PAIRED COMPARISONS BETWEen SPARSE CODING SR (SCSR), BM3 D + SCSR, SCSR + BM3 D AND THE PROPOSED JOINT SCSR + Deblocking

\begin{tabular}{cccccc}
\hline & $\begin{array}{c}\text { Proposed } \\
\text { SCSR }\end{array}$ & Cascade I & Cascade II & SCSR & Average \\
\hline Proposed SCSR & - & $\begin{array}{c}\mathbf{1 2 7} \\
\mathbf{( 7 1 \% )}\end{array}$ & $\begin{array}{c}\mathbf{1 4 8} \\
\mathbf{( 8 2} \%)\end{array}$ & $\begin{array}{c}\mathbf{1 7 0} \\
\mathbf{( 9 4 \% )}\end{array}$ & $\mathbf{8 2 . 4 \%}$ \\
\hline $\begin{array}{c}\text { Cascade I } \\
\text { (SCSR+BM3D) }\end{array}$ & $\begin{array}{c}53 \\
(29 \%)\end{array}$ & - & $\begin{array}{c}91 \\
(51 \%)\end{array}$ & $\begin{array}{c}147 \\
(82 \%)\end{array}$ & $53.9 \%$ \\
\hline $\begin{array}{c}\text { Cascade II } \\
\text { BM3D+SCSR) }\end{array}$ & $\begin{array}{c}32 \\
(18 \%)\end{array}$ & $\begin{array}{c}89 \\
(49 \%)\end{array}$ & - & $\begin{array}{c}143 \\
(79 \%)\end{array}$ & $48.9 \%$ \\
\hline SCSR & $\begin{array}{c}10 \\
(6 \%)\end{array}$ & $\begin{array}{c}33 \\
(18 \%)\end{array}$ & $\begin{array}{c}37 \\
(21 \%)\end{array}$ & - & $14.8 \%$ \\
\hline
\end{tabular}

TABLE V

WinNing Frequency Matrix of SubJeCtive PaIRED COMPARISONS BETWEen SCSR, SELFSR, THE PROPOSED JOINT SCSR + Deblocking, AND JOINT SelfSR + Deblocking

\begin{tabular}{cccccc}
\hline & $\begin{array}{c}\text { Proposed } \\
\text { SelfSR }\end{array}$ & $\begin{array}{c}\text { Proposed } \\
\text { SCSR }\end{array}$ & SelfSR & SCSR & Average \\
\hline $\begin{array}{c}\text { Proposed } \\
\text { SelfSR }\end{array}$ & - & $\begin{array}{c}\mathbf{1 1 7} \\
(\mathbf{6 5} \%)\end{array}$ & $\begin{array}{c}\mathbf{1 6 8} \\
\mathbf{( 9 3 \% )}\end{array}$ & $\begin{array}{c}\mathbf{1 7 1} \\
\mathbf{( 9 5 \% )}\end{array}$ & $\mathbf{8 4 . 4 \%}$ \\
\hline $\begin{array}{c}\text { Proposed } \\
\text { SCSR }\end{array}$ & $\begin{array}{c}63 \\
(35 \%)\end{array}$ & - & $\begin{array}{c}159 \\
(88 \%)\end{array}$ & $\begin{array}{c}170 \\
(94 \%)\end{array}$ & $72.69 \%$ \\
\hline SelfSR & $\begin{array}{c}12 \\
(7 \%)\end{array}$ & $\begin{array}{c}21 \\
(12 \%)\end{array}$ & - & $\begin{array}{c}100 \\
(56 \%)\end{array}$ & $24.6 \%$ \\
\hline SCSR & 9 & 10 & 80 & - & $18.3 \%$ \\
\hline
\end{tabular}

learned characteristics to further partition an additional ringing sub-dictionary in the structure shown in Fig. 3(g). However, our experimental results show that, although the proposed framework can still mitigate ringing artifacts to some extent, the de-ringing performance is not satisfactory. Fig. 10 illustrates an example of ringing artifacts removal based on the proposed framework where Fig. 10(a) shows an image after joint SR and deblocking (without de-ringing) and Fig. 10(b) shows the version after joint SR, deblocking and de-ringing using the proposed framework. We can observe from Fig. 10(b) that the ringing artifacts, although being less severe compared to Fig. 10(a), are still clearly visible. One of the main reasons is that the structure of ringing artifact is usually irregular. Hence, it is not easy to well capture the characteristics of ringing artifact by the HoG features and automatically self-learn a pair of dictionaries of ringing atoms. Besides, ringing detection is not accurate enough, making it difficult to extract enough good exemplar patches for learning. To achieve better visual quality of a reconstructed image, performing a post-processing step to remove the ringing artifacts might be a choice, which would, however, also blur some image details, as exemplified in Fig. 10(c). For future work, integrating de-ringing into our SR framework to achieve satisfactory de-ringing performance still needs further study.

\section{Computational Complexity}

The proposed method was implemented in 32-bit MATLAB on a personal computer equipped with Intel Core i7-4470 processor and 32 GB memory. Table VI lists the run-time of individual steps, including the dictionary learning and sparse reconstruction, for the test image shown in Fig. 7 based on the SelfSR 


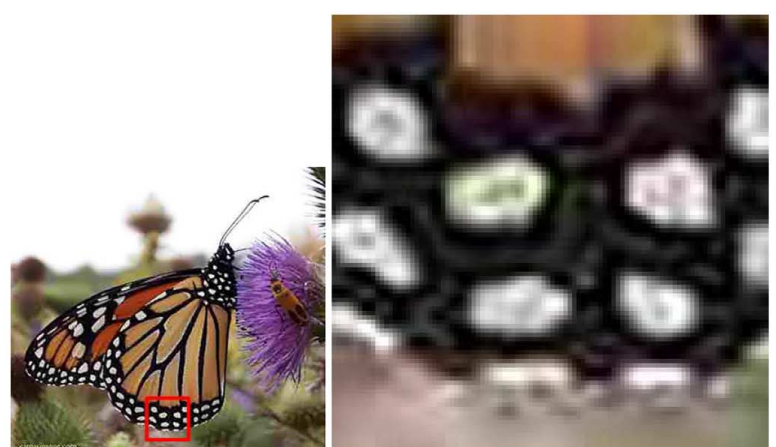

(a)

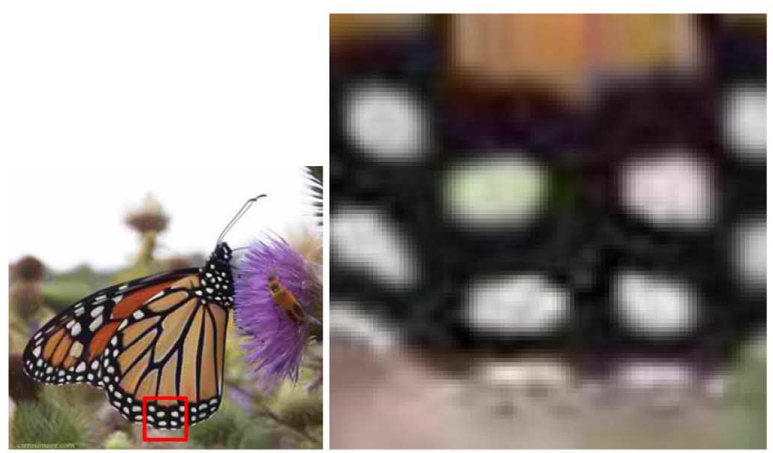

(b)

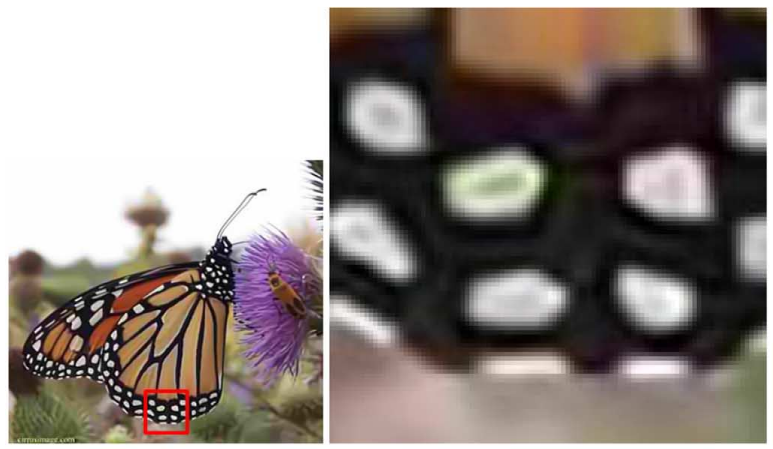

(c)

Fig. 10. SR results obtained by (a) the proposed method without de-ringing; (b) the proposedSR + Deblocking + Deringing method; and (c) the proposed SR+Deblocking method followed by BM3D filtering [33] to remove ringing artifacts (with some details blurred).

TABLE VI

RUN-Time (IN SECONDS) OF THE DictionARY LEARNING AND Sparse Reconstruction Phases in the SelfSR Method AND the PROPOSED SELFSR Method

\begin{tabular}{cccc}
\hline Evaluated methods & $\begin{array}{c}\text { Dictionary } \\
\text { learning }\end{array}$ & $\begin{array}{c}\text { Sparse } \\
\text { reconstruction }\end{array}$ & Total time \\
\hline SelfSR & $229.14 \mathrm{~s}$ & $31.32 \mathrm{~s}$ & $260.46 \mathrm{~s}$ \\
Proposed SelfSR & $123.64 \mathrm{~s}$ & $30.10 \mathrm{~s}$ & $153.74 \mathrm{~s}$ \\
\hline
\end{tabular}

approach and the proposed SelfSR method, respectively. In the dictionary learning, our method learns an additional pair of dictionaries (either for training patches with or without blocking artifacts separately), whereas the SelfSR method learns only one pair of dictionaries. In our implementation, the size of each dictionary pair is set to 512 (total 1024 in size of two pairs of dictionary). The computational complexity for learning each dictio-
TABLE VII

Run-TIME (IN SECONDS) OF THE DICTIONARY LEARNING AND SPARSE RECONSTRUCTION PHASES IN THE SCSR METHOD AND THE PROPOSED SCSR METHOD

\begin{tabular}{ccc}
\hline Evaluated methods & $\begin{array}{c}\text { Dictionary learning } \\
\text { (Offline) }\end{array}$ & $\begin{array}{c}\text { Sparse } \\
\text { reconstruction }\end{array}$ \\
\hline SCSR & $45541 \mathrm{~s}$ & $123.7 \mathrm{~s}$ \\
Proposed SCSR & $42331 \mathrm{~s}$ & $121.9 \mathrm{~s}$ \\
\hline
\end{tabular}

nary pair of size, 512 , is significantly lower than that for learning a dictionary pair of size, 1024, used in the SelfSR method. Based on Table VI, the complexity of sequentially learning two pairs of dictionaries in our method is still lower than that for learning a single dictionary pair of size 1024 used in the SelfSR method. Moreover, the complexity of our method can be further reduced by learning two pairs of dictionaries in parallel should a multicore processor be used.

On the other hand, the complexity of sparse reconstruction phase mainly relies on the dictionary size and the number of non-zero coefficients to be solved which is set to be the same for all evaluated dictionary learning-based methods. Similarly, the complexity of the sparse reconstruction required for the two pairs of learned dictionaries of size 512 for each in our method is lower than that of the SelfSR method with a dictionary size of 1024. The sparse reconstruction operations with respect to the two dictionary pairs in our method can be also performed in parallel to further reduce the complexity.

Table VII compares the run-time complexities of the SCSR and the proposed SCSR + deblocking methods. Both the two methods need to train dictionaries from an external training dataset. Therefore, their dictionary leaning is an offline process which consumes significantly higher complexity than that of SelfSR-based schemes, whereas the sparse reconstruction is performed on-line. Table VII shows that our method is still of lower time complexity, compared to the original SCSR.

The other overheads in the learning phase of our methods include the blocking artifact detection and dictionary partitioning, whose complexities are usually insignificant. By properly fusing the cores of the SR and deblocking operations into a unified MCA-based framework, the proposed framework can simultaneously achieve both SR and deblocking while reducing computational complexity, compared with a pure learning-based SR scheme. Moreover, the computational complexity of our method is obviously lower than that of cascading-based approach, if a similar SR scheme is used.

\section{CONCLUSION}

In this paper, we proposed a learning-based SR framework to achieve joint single-image SR and deblocking for image sparse representation for modeling the relationship between LR and HR image patches in terms of the learned dictionaries, respectively, for image patches with and without blocking artifacts. As a result, image SR and deblocking can be simultaneously achieved via sparse representation and MCA-based image decomposition. Our experimental results demonstrate the efficacy of the proposed algorithm on self-learning SR and sparse-coding SR. Furthermore, our method can be naturally 
extended to remove other types of noise or structured patterns while performing SR for images.

\section{REFERENCES}

[1] M. Y. Shen and C. C. J. Kuo, "Review of postprocessing techniques for compression artifacts removal," J. Vis. Commun. Image Rep., vol. 9, no. 1, pp. 2-14, Mar. 1998.

[2] S. C. Park, M. K. Park, and M. G. Kang, "Super-resolution image reconstruction: A technical overview," IEEE Signal Process. Mag., vol. 20, no. 3, pp. 21-36, May 2003.

[3] S. Farsiu, M. Robinson, M. Elad, and P. Milanfar, "Fast and robust multiframe super resolution," IEEE Trans. Image Process., vol. 13, no. 10, pp. 1327-1344, Oct. 2004.

[4] H. S. Hou and H. C. Andrews, "Cubic splines for image interpolation and digital filtering," IEEE Trans. Signal Process., vol. 26, no. 6, pp. 508-517, Dec. 1978.

[5] W. T. Freeman, T. R. Jones, and E. C. Pasztor, "Example-based superresolution," IEEE Comput. Graph. Appl., vol. 22, no. 2, pp. 56-65, Mar./Apr. 2002.

[6] D. Glasner, S. Bagon, and M. Irani, "Super-resolution from a single image," in Proc. IEEE Int. Conf. Comput. Vis., Kyoto, Japan, Sep. 2009, pp. 349-356.

[7] G. Freedman and R. Fattal, "Image and video upscaling from local self-examples," ACM Trans. Graph., vol. 30, no. 2, pp. 12.1-12.11, 2011.

[8] C.-C. Hsu, L.-W. Kang, and C.-W. Lin, "Temporally coherent super-resolution of textured video via dynamic texture synthesis," IEEE Trans. Image Process., vol. 24, no. 3, pp. 919-931, Mar. 2015.

[9] J. Yang, J. Wright, T. Huang, and Y. Ma, "Image super-resolution via sparse representation," IEEE Trans. Image Process., vol. 19, no. 11, pp. 2861-2873, Nov. 2010.

[10] J. Yang, Z. Wang, Z. Lin, S. Cohen, and T. S. Huang, "Coupled dictionary training for image super-resolution," IEEE Trans. Image Process., vol. 21, no. 8, pp. 3467-3478, Aug. 2012.

[11] W. Dong, L. Zhang, G. Shi, and X. Wu, "Image deblurring and superresolution by adaptive sparse domain selection and adaptive regularization," IEEE Trans. Image Process., vol. 20, no. 7, pp. 1838-1857, Jul. 2011.

[12] J. Ren, J. Liu, and Z. Guo, "Context-aware sparse decomposition for image denoising and super-resolution," IEEE Trans. Image Process., vol. 22, no. 4, pp. 1456-1469, Apr. 2013.

[13] C.-Y. Yang, J.-B. Huang, and M.-H. Yang, "Exploiting self-similarities for single frame super-resolution," in Proc. Asian Conf. Comput. Vis., Queenstown, New Zealand, Nov. 2010, pp. 497-510.

[14] M.-C. Yang and Y.-C. F. Wang, "A self-learning approach to single image super-resolution," IEEE Trans. Multimedia, vol. 15, no. 3, pp. 498-508, Apr. 2013.

[15] Z. Xiong, X. Sun, and F. Wu, "Robust web image/video super-resolution," IEEE Trans. Image Process., vol. 19, no. 8, pp. 2017-2028, Aug. 2010.

[16] T. Q. Pham, L. J. van Vliet, and K. Schutte, "Resolution enhancement of low quality videos using a high-resolution frame," in Proc. SPIE, San Jose, CA, USA, Jan. 2006, vol. 6077, pp. 607708-1-607708-10.

[17] F. Liu, J. Wang, S. Zhu, M. Gleicher, and Y. Gong, "Noisy video superresolution," in Proc. ACM Multimedia, Vancouver, Canada, Oct. 2008, pp. 713-716.

[18] T. Yamaguchi, H. Fukuda, R. Furukawa, H. Kawasaki, and P. F. Sturm, "Video deblurring and super-resolution technique for multiple moving objects," in Proc. Asian Conf. Comput. Vis., Queenstown, New Zealand, Nov. 2010, pp. 127-140.

[19] C. Liu and D. Sun, "A bayesian approach to adaptive video super resolution," in Proc. IEEE Conf. Comput. Vis. Pattern Recog., Jun. 2011, pp. 209-216.

[20] G. A. Triantafyllidis, D. Tzovaras, and M. G. Strintzis, "Blocking artifact detection and reduction in compressed data," IEEE Trans. Circuits Syst. Video Technol., vol. 12, no. 10, pp. 877-890, Oct. 2002.

[21] P. List, A. Joch, J. Lainema, G. Bjontegaard, and M. Karczewicz, "Adaptive deblocking filter," IEEE Trans. Circuits Syst. Video Technol., vol. 13, no. 7, pp. 614-619, Jul. 2003.

[22] C.-H. Yeh, Y.-W. Chiou, L.-W. Kang, C.-W. Lin, and S.-J. Fan-Jiang, "Self-learning-based post-processing for image/video deblocking via sparse representation," J. Vis. Commun. Image Represent., vol. 25, no. 5, pp. 891-903, Jul. 2014.
[23] L.-W. Kang, B.-C. Chuang, C.-C. Hsu, C.-W. Lin, and C.-H. Yeh, "Self-learning-based single image super-resolution of a highly compressed image," in Proc. IEEE Workshop Multimedia Signal Process., Sardinia, Italy, Sep. 2013, pp. 224-229.

[24] G. K. Wallace, "The JPEG still picture compression standard," Commun. ACM, vol. 34, no. 4, pp. 30-44, Apr. 1991.

[25] T. Wiegand, G. J. Sullivan, G. Bjntegaard, and A. Luthra, "Overview of the H.264/AVC video coding standard," IEEE Trans. Circuits Syst. Video Technol., vol. 13, no. 7, pp. 560-576, Jul. 2003.

[26] G. J. Sullivan, J.-R. Ohm, W. Han, and T. Wiegand, "Overview of the high efficiency video coding (HEVC) Standard," IEEE Trans. Circuits Syst. Video Technol., vol. 22, no. 12, pp. 1649-1668, Dec. 2012.

[27] J. L. Starck, M. Elad, and D. L. Donoho, "Image decomposition via the combination of sparse representations and a variational approach," IEEE Trans. Image Process., vol. 14, no. 10, pp. 1570-1582, Oct. 2005

[28] J. M. Fadili, J. L. Starck, J. Bobin, and Y. Moudden, "Image decomposition and separation using sparse representations: An overview," Proc. IEEE, vol. 98, no. 6, pp. 983-994, Jun. 2010.

[29] L.-W. Kang, C.-W. Lin, and Y.-H. Fu, "Automatic single-image-based rain streaks removal via image decomposition," IEEE Trans. Image Process., vol. 21, no. 4, pp. 1742-1755, Apr. 2012.

[30] D.-A. Huang, L.-W. Kang, Y.-C. F. Wang, and C.-W. Lin, "Self-learning based image decomposition with applications to single image denoising," IEEE Trans. Multimedia, vol. 16, no. 1, pp. 83-93, Jan. 2014.

[31] B. A. Olshausen and D. J. Field, "Emergence of simple-cell receptive field properties by learning a sparse code for natural images," Nature, vol. 381 , no. 13 , pp. 607-609, Jun. 1996.

[32] J. Mairal, F. Bach, J. Ponce, and G. Sapiro, "Online learning for matrix factorization and sparse coding," J. Mach. Learn. Res., vol. 11, pp. 19-60, 2010.

[33] K. Dabov, A. Foi, V. Katkovnik, and K. Egiazarian, "Image denoising by sparse 3D transform-domain collaborative filtering," IEEE Trans. Image Process., vol. 16, no. 8, pp. 2080-2095, Aug. 2007.

[34] N. Dalal and B. Triggs, "Histograms of oriented gradients for human detection," in Proc. IEEE Conf. Comput. Vis. Pattern Recog., San Diego, CA, USA, Jun. 2005, pp. 886-893.

[35] M. Elad and M. Aharon, "Image denoising via sparse and redundant representations over learned dictionaries," IEEE Trans. Image Process., vol. 15, no. 12, pp. 3736-3745, Dec. 2006.

[36] H. Lim and H. W. Park, "A ringing-artifact reduction method for blockDCT-based image resizing," IEEE Trans. Circuits Syst. Video Technol., vol. 21 , no. 7 , pp. 879-889, Jul. 2011.

[37] C.-C. Hsu, C.-W. Lin, Y. Fang, and W. Lin, "Objective quality assessment for image retargeting based on perceptual geometric distortion and information loss," IEEE J. Sel. Topics Signal Process., vol. 8, no. 3, pp. 377-389, Jun. 2014.

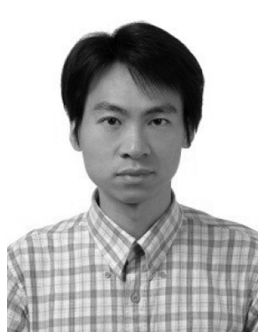

Li-Wei Kang (S'04-A'05-M'06) received the B.S., M.S., and Ph.D. degrees in computer science from National Chung Cheng University, Chiayi, Taiwan, in 1997, 1999, and 2005, respectively.

Since February 2013, he has been an Assistant Professor with the Graduate School of Engineering Science and Technology, Doctoral Program and the Department of Computer Science and Information Engineering, National Yunlin University of Science and Technology, Yunlin, Taiwan. Before that, he worked as a Postdoctoral Research Fellow from 2005 to 2010 and as an Assistant Research Scholar from 2010 to 2013 with the Institute of Information Science, Academia Sinica, Taipei, Taiwan. His research interests include multimedia content analysis and multimedia communications.

Dr. Kang served as the Editor-in-Chief of the Gate to Multimedia Processing (Science Gate Publishing). He served on the editorial board of the International Journal of Distributed Sensor Networks, an Editorial Advisory Board Member of the book Visual Information Processing in Wireless Sensor Networks: Technology, Trends and Applications (IGI Global, 2011), a Guest Editor of the International Journal of Electrical Engineering (Taiwan), Special Session Co-Chair of APSIPA ASC 2012, and Registration Co-Chair of APSIPA ASC 2013. He serves as Demo/Exhibition Co-Chair of IEEE ICCE-TW 2015. He was the recipient of a Top 10\% Paper Award at IEEE MMSP 2013. 


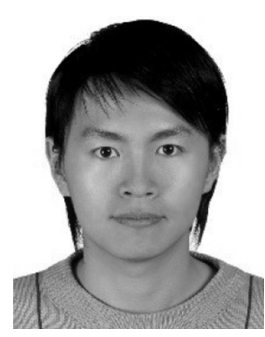

Chih-Chung Hsu received the B.S. degree in information management from Ling-Tung University of Science and Technology, Taichung, Taiwan, in 2004, the M.S. degree in electrical engineering from National Yunlin University of Science and Technology, Douliu, Taiwan, in 2007, and the Ph.D. degree in electrical engineering from National Tsing Hua University (NTHU), Hsinchu, Taiwan, in 2014.

$\mathrm{He}$ is a Postdoctoral Researcher with the Institute of Communications Engineering, NTHU. His research interests include computer vision and image

and video processing

Dr. Hsu was the recipient of a Top 10\% Paper Award at IEEE MMSP 2013.

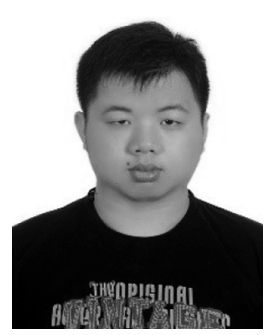

Boqi Zhuang received the B.S. degree in communication engineering from National Central University, Taoyuan, Taiwan, in 2011, and the M.S. degree in electrical engineering from National Tsing Hua University, Hsinchu, Taiwan, in 2013.

Since August 2013, he has been a Software Engineer with Altek Corporation, Hsinchu, Taiwan. His research interests include image/video processing and compression.

Mr. Zhuang was the recipient of a Top 10\% Paper Award at IEEE MMSP 2013.

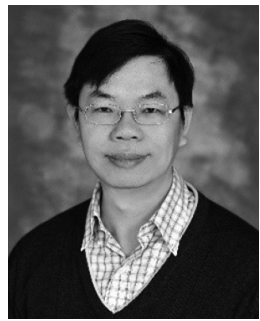

Chia-Wen Lin (S'95-M'00-SM'04) received the $\mathrm{Ph} . \mathrm{D}$. degree in electrical engineering from National Tsing Hua University (NTHU), Hsinchu, Taiwan, in 2000.

He was with the Information and Communications Research Labs, Industrial Technology Research Institute, Hsinchu, Taiwan, from 1992 to 2000 . He was with the Department of Computer Science and Information Engineering, National Chung Cheng University, Min-Hsiung, Taiwan, from 2000 to 2007 . He is currently a Professor with the Department of Elec- trical Engineering and the Institute of Communications Engineering, NTHU. $\mathrm{He}$ is also an Adjunct Professor with the Department of Computer Science and Information Engineering, Asia University, Taichung, Taiwan. His research interests include image/video processing and video networking.

Dr. Lin has served as an Associate Editor of the IEEE TRANSACTIONS ON Circuits AND SYSTEMS FOR VIDEO TECHNOLOGY, the IEEE TRANSACTIONS ON Multimedia, the IEEE MultiMedia Magazine, and the Journal of Visual Communication and Image Representation. He also serves as a Steering Committee Member of the IEEE TRANSACTIONS ON Multimedia. He is currently Chair of the Multimedia Systems and Applications Technical Committee of the IEEE Circuits and Systems Society. He served as Technical Program Co-Chair of the IEEE ICME in 2010 and Special Session Co-Chair of IEEE ICME in 2009. He is a Distinguished Lecturer of the Asia-Pacific Signal and Information Processing Association. His paper was the recipient of the Young Investigator Award presented by VCIP 2005. He was the recipient of the Young Investigator Awards presented by National Science Council, Taiwan, in 2006.

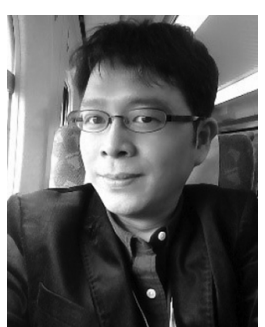

Chia-Hung Yeh (M'03-SM'12) received the B.S. and $\mathrm{Ph} . \mathrm{D}$. degrees in electrical engineering from National Chung Cheng University, Min-Hsiung, Taiwan, in 1997 and 2002, respectively.

He joined the Department of Electrical Engineering, National Sun Yat-sen University (NSYSU), Kaohsiung, Taiwan, in 2007, where he is currently a Professor. He has coauthored more than 150 technical international conferences and journal papers, and holds 42 patents in the U.S., Taiwan, and China. His research interests include multimedia communication and multimedia signal processing.

Dr. Yeh serves on the Editorial Board of the Journal of Visual Communication and Image Representation and the EURASIP Journal on Advances in Signal Processing. In addition, he has organized several international/domestic conferences, serving as keynote speaker, session chair, and technical program committee and program committee member. He was the recipient of the 2007 Young Researcher Award from NSYSU, the 2011 Distinguished Young Engineer Award from the Chinese Institute of Electrical Engineering, the 2013 Distinguished Young Researcher Award from NSYSU, and the 2013 IEEE MMSP Top 10\% Paper Award. 